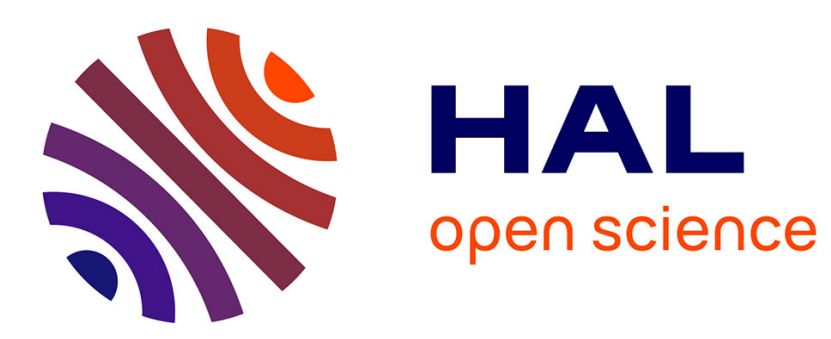

\title{
AC measurement of heat capacity and magnetocaloric effect for pulsed magnetic fields
}

\author{
Yoshimitsu Kohama, C. Marcenat, Thierry Klein, Marcelo Jaime
}

\section{To cite this version:}

Yoshimitsu Kohama, C. Marcenat, Thierry Klein, Marcelo Jaime. AC measurement of heat capacity and magnetocaloric effect for pulsed magnetic fields. Review of Scientific Instruments, 2010, 81, pp.104902. 10.1063/1.3475155 . hal-00957185

\section{HAL Id: hal-00957185 \\ https://hal.science/hal-00957185}

Submitted on 10 Mar 2014

HAL is a multi-disciplinary open access archive for the deposit and dissemination of scientific research documents, whether they are published or not. The documents may come from teaching and research institutions in France or abroad, or from public or private research centers.
L'archive ouverte pluridisciplinaire HAL, est destinée au dépôt et à la diffusion de documents scientifiques de niveau recherche, publiés ou non, émanant des établissements d'enseignement et de recherche français ou étrangers, des laboratoires publics ou privés. 


\title{
AC measurement of heat capacity and magnetocaloric effect for pulsed magnetic fields
}

\author{
Yoshimitsu Kohama, ${ }^{1, a)}$ Christophe Marcenat, ${ }^{2}$ Thierry Klein, ${ }^{3}$ and Marcelo Jaime ${ }^{1}$ \\ ${ }^{1}$ MPA-CMMS, Los Alamos National Laboratory, Los Alamos, New Mexico 87545, USA \\ ${ }^{2}$ CEA-INAC/UJF-Grenoble 1, SPSMS, UMR-E 9001, 17 rue des Martyrs, Grenoble 38054, France \\ ${ }^{3}$ Institut Néel, CNRS, BP 166, Grenoble 38042, France; Institut Universitaire de France, BP 53, \\ Grenoble 38041, France; and Universitè Joseph Fourier, BP 53, Grenoble 38041, France
}

(Received 16 March 2010; accepted 14 July 2010; published online 15 October 2010)

\begin{abstract}
A new calorimeter for measurements of the heat capacity and magnetocaloric effect of small samples in pulsed magnetic fields is discussed for the exploration of thermal and thermodynamic properties at temperatures down to $2 \mathrm{~K}$. We tested the method up to $\mu_{0} H=50 \mathrm{~T}$, but it could be extended to higher fields. For these measurements we used carefully calibrated bare-chip Cernox ${ }^{\circledR}$ and $\mathrm{RuO}_{2}$ thermometers, and we present a comparison of their performances. The monotonic temperature and magnetic field dependences of the magnetoresistance of $\mathrm{RuO}_{2}$ allow thermometry with a precision as good as $\pm 4 \mathrm{mK}$ at $T=2 \mathrm{~K}$. To test the performance of our calorimeter, heat capacity and magnetocaloric effect for the spin-dimer compound $\mathrm{Sr}_{3} \mathrm{Cr}_{2} \mathrm{O}_{8}$ and the triangular lattice antiferromagnet $\mathrm{RbFe}\left(\mathrm{MoO}_{4}\right)_{2}$ are presented. (C) 2010 American Institute of Physics.
\end{abstract}

[doi:10.1063/1.3475155]

\section{INTRODUCTION}

Thermal properties such as specific heat and entropy are fundamental material properties that help us understand the most relevant microscopic mechanisms. Among them, the quick and reliable determination of magnetic fieldtemperature $(H, T)$ phase diagrams is extremely valuable in condensed matter physics since it helps establish the basic ingredients for minimalistic modeling and prediction. Many thermodynamic studies including specific heat $(C)$ and magnetocaloric effect (MCE) in very high magnetic fields have been reported over the past decade. ${ }^{1-13}$ However, these reports have all been limited to the measurements carried out in dc magnets, with the exception of one pulsed magnet having an unusually large ( $\sim 500 \mathrm{~ms})$ magnetic field plateau.

The Pulsed Magnetic Field Facility of the National High Magnetic Field Laboratory at Los Alamos National Laboratory currently provides pulsed magnetic fields up to $85 \mathrm{~T}$ of various durations, ranging from 0.025 to $2 \mathrm{~s}$, with and without flat tops. These magnets are world-class tools that so far have yet to be fully utilized for thermal studies of materials at low temperatures. Here we describe the development of a method for measuring heat capacity in pulsed magnetic fields using ac calorimetry techniques. Since this method was introduced in 1968 by Sullivan and Seidel, ${ }^{14}$ many studies in organic crystals, ${ }^{15}$ superconductor, ${ }^{16-18}$ liquid crystals, ${ }^{19}$ fluids, $^{20}$ and biological materials ${ }^{21,22}$ have been carried out. In particular, the application of ac calorimetry techniques to measurements under pressure stimulated intense research. ${ }^{23-25}$ This arises from the fact that the ac calorimetry technique exploits effective noise rejection strategies and presents a very high sensitivity. Hence, the appli-

\footnotetext{
${ }^{a)}$ Electronic mail: ykohama@lanl.gov.
}

cation of the ac calorimetry for measurements in the demanding electromagnetic environment of high/fast pulsed magnetic fields provides a new avenue for high quality thermal and thermodynamic materials research.

In addition to heat capacity, our calorimeter can also be used to measure the MCE to map out the full $(H, T)$ phase diagram in an efficient way, i.e., revealing phase boundaries before an extensive $C(H, T)$ experiment is run, and to directly quantify entropy changes at phase boundaries. To assess the potential and performance of this method, new results are compared with MCE and $C_{p}$ data recently obtained up to $\mu_{0} H=35 \mathrm{~T}$ in a dc resistive magnet in the spin dimmer compound $\mathrm{Sr}_{3} \mathrm{Cr}_{2} \mathrm{O}_{8}$ and the triangular lattice antiferromagnet $\mathrm{RbFe}\left(\mathrm{MoO}_{4}\right)_{2}$. To the best of our knowledge, this is the first report of heat capacity and MCE measurements in $250 \mathrm{~ms}$ pulsed magnetic fields.

\section{PRINCIPLES UNDERLYING HEAT CAPACITY AND MCE MEASUREMENTS}

Heat capacity is a fundamental thermodynamic quantity, which is defined as the amount of energy or heat $(\delta q)$ required to increase the temperature of a material by the amount $(\delta T), C=\delta q / \delta T$. Using the expression of entropy $(S)$ valid for a reversible process, $T d S=\delta q$, the specific heat can be expressed as $C_{i}=T(\partial S / \partial T)_{i}$, where the index $i$ refers to the thermodynamic quantities kept constant during the experiment such as pressure $p$, volume $V$, and magnetic field $H$. Thus, the magnetic contribution to the heat capacity of materials measures the change of the spin entropy as a function of temperature. It is known that the spin entropy of magnetic materials can be altered with applied external magnetic fields. The change in the spin entropy as a function of magnetic fields leads to a measurable MCE, which is generally recognized as the change in temperature through the 

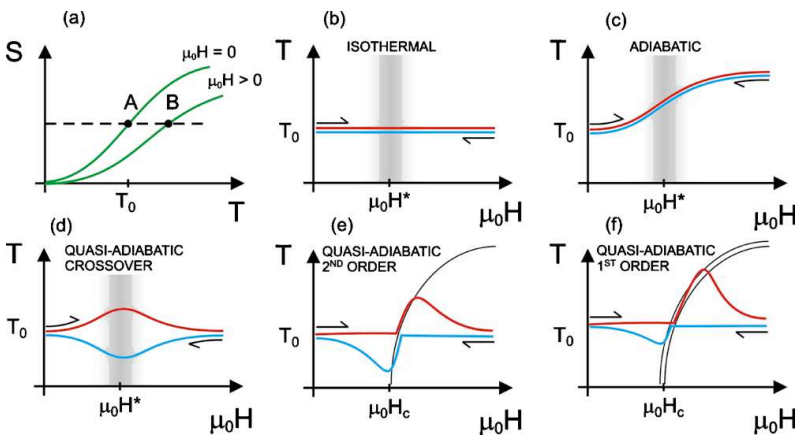

FIG. 1. (Color online) (a) Schematic entropy $(S)$ vs temperature $(T)$ in a hypothetical system where the entropy is reduced by an applied magnetic field as is observed in a paramagnet. (b) Temperature of our hypothetical sample when the magnetic field is changed in an isothermal fashion, allowing the full exchange of heat with the bath. (c) The same field sweep done in an adiabatic fashion. As the sample cannot exchange heat with the bath the temperature changes reversibly as indicated in (a) when the sample travels from A to B and back to A. (d) The most realistic case of quasiadiabatic field sweep reveals changes in the sample temperature that permit the determination of the relevant field/energy scale of the sample under study. (e) When the quasiadiabatic field sweep is performed in the presence of a fieldinduced second order phase transition at the critical field $\mu_{0} H_{\mathrm{c}}$, the magnetocaloric effect reveals sharp features. (f) First order phase transitions where dissipative mechanisms are present show characteristic asymmetry due to the release of heat in both directions of the magnetic field sweep.

application or removal of an external magnetic field. Using the general formula for the entropy change in temperature and magnetic field, one obtains

$$
\delta q=T d S=T\left(\frac{\partial S}{\partial T}\right)_{H} d T+T\left(\frac{\partial S}{\partial H}\right)_{T} d H .
$$

This equation can be rearranged as

$$
P=C \frac{d T}{d t}+T\left(\frac{\partial S}{\partial H}\right) \frac{d H}{T},
$$

where $P=\delta q / d t$ is the power applied to the system. The second term on the right hand side represents the power generated by the entropy change in field, which leads to a MCE.

The essence of MCE is depicted in Fig. 1. Indeed, Fig. 1(a) shows the entropy versus temperature for a magnetic system in zero magnetic field and in a finite applied magnetic field. The nature of the example material is arbitrary, with the only requirement being that the system entropy is reduced $(\partial S / \partial H<0)$ by the application of a magnetic field. When we increase the applied magnetic field in adiabatic conditions, as indicated by the dashed line in Fig. 1(a), the sample temperature must increase from A to B. When the magnetic field is decreased back to zero in the same adiabatic conditions, the sample temperature decreases from B to A. The previous statements are true, disregarding the presence or absence of a field-induced phase transition. Note that in the cases where the entropy of the system increases with the applied magnetic field, such as when charge, spin, or crystal field energy gaps are closed, the sign of the temperature change is simply inverted. Depending on the sweep rate of the magnetic field $d H / d t$ and the strength of the thermal link between the sample and the thermal bath during the MCE experiment, three distinct situations are possible. Using the thermal conductance of the thermal link $\kappa, P$ can be expressed as the heat transferred from the thermal bath $P=\kappa\left(T_{\text {bath }}-T\right)$ and we can introduce a general formula for MCE measurements,

$$
\kappa\left(T_{\text {bath }}-T\right)=\kappa \tau_{1} \frac{d T}{d H} \frac{d H}{d t}+T\left(\frac{\partial S}{\partial H}\right)_{T} \frac{d H}{d t},
$$

where $\tau_{1}$ is called the external time constant, $\tau_{1}=C / \kappa$. If $d H / d t$ is extremely slow compared to $\tau_{1}$ and/or $\kappa$ is large, the experiment is performed in isothermal conditions $\left(T-T_{\text {bath }} \cong 0\right)$, and no changes in the sample temperature versus magnetic field are observed as shown in Fig. 1(b), where the arrows represent the sweep direction of the magnetic field. On the other hand, if the experiment is performed in adiabatic conditions $(d H / d t$ is large and/or $\kappa$ is small), the sample temperature changes during the field sweep [see Fig. 1(c)]. In this case the sample temperature increases during the up-sweep, in agreement with Fig. 1(a), and decreases during the down-sweep. The temperature change of the sample in this case is completely reversible. Figure 1(d) displays the most common practical case, where the experiment is performed in quasiadiabatic conditions $(d H / d t$ and/or $\kappa$ are intermediate values). Here the sample overheats during the field up-sweep and then returns to the bath temperature within a time comparable to $\tau_{1}$. Since it overcools during the field down-sweep, an open loop structure in MCE is observed. This last experiment is the most useful of all three limits to determine energy scale crossovers. In the typical MCE experiments in dc fields $\left(\tau_{1} \sim 1 \mathrm{~s}, d H / d t \sim 0.01 \mathrm{~T} / \mathrm{s}\right.$, $d T / d H \sim 0.1 \mathrm{~K} / \mathrm{T}$, and $\left.T-T_{\text {bath }} \sim 0.1 \mathrm{~K}\right),{ }^{4-7,15}$ the contribution of the first term on the right hand side of Eq. (3) is much smaller than the term on the left-hand side, hence it can be ignored. The resulting formula allows us to evaluate the change of the entropy as a function of magnetic field. ${ }^{26}$

The effects in the MCE are greatly enhanced when the applied magnetic field induces phase transitions, disregarding the order of the phase transition. This phenomenon is particularly pronounced at temperatures and fields corresponding to magnetic phase transitions, schematically described in Figs. 1(e) and 1(f), and thus it is a powerful tool for the investigation of the magnetic state and mechanisms of these magnetic transitions. ${ }^{8,9,11-13,26}$ The shape of the magnetocaloric response depends on the absence/existence of dissipative processes at crossover fields or phase boundaries [reversible phase transitions in Figs. 1(c)-1(e)]. If an ideal reversible phase transition occurs, a symmetric temperature change in the up- and down-sweeps such as in Figs. 1(d) and $1(\mathrm{e})$ is observed. However, if dissipative processes take place, such as domain wall motion, nonsymmetric temperature changes can be observed as different magnitudes of the temperature changes during up-sweep and down-sweep as schematically displayed in Fig. 1(f). The MCE data can then be used to separate reversible from irreversible processes at phase boundaries in magnetic materials, although making a clear distinction between transitions of different orders is much more difficult. ${ }^{9}$ Contrary to the case of properties measured as a function of temperature, hysteresis in MCE measured versus field is not (as clearly seen in the qualitative figures) a measurement of latent heat, and hence not direct 
evidence for a first order phase transition. Sharpness of the MCE anomaly, in a similar way, can be varied with $d H / d t$, $\tau_{1}, \kappa$, and so on, and is not an indication of a first order phase transition, as often suggested. One property that MCE reveals unambiguously is the presence or absence of dissipation/irreversibility at the phase boundary, which can sometimes be linked to the order of the phase transition. ${ }^{9}$

In the heat capacity measurements, $P$ in Eq. (2) contains the term of the applied ac power to the heater, $P=\kappa\left(T_{\text {bath }}-T\right)+P_{\text {ac }}[1+\cos (2 \omega t)]$, where the $P_{\text {ac }}$ is generated by an ac current of frequency $\omega$ and the sample temperature is $T=T_{\text {bath }}+T_{\mathrm{dc}}+\left|T_{\mathrm{ac}}\right| \cos (2 \omega t+\phi)$. Substituting $P$ and $T$ into Eq. (2) gives the dc and ac components

$$
T_{\mathrm{dc}}=\frac{P_{\mathrm{ac}}-T_{\mathrm{bath}}\left(\frac{\partial S}{\partial H}\right)_{T} \frac{d H}{d T}}{\kappa+\left(\frac{\partial S}{\partial H}\right)_{T} \frac{d H}{d T}},
$$

which simplifies to the usual case when $d H / d t=0$ : $T_{\mathrm{dc}}=P_{\mathrm{ac}} / \kappa$,

$$
T_{\mathrm{ac}}=\left|T_{\mathrm{ac}}\right| \exp (i \varphi)=\frac{P_{\mathrm{ac}}}{\kappa+\left(\frac{\partial S}{\partial H}\right) \frac{d H}{d t}+i 2 \omega C},
$$

where the phase difference between the temperature oscillation and the periodic heating power is

$$
\tan (\varphi)=-2 \omega C / \kappa+\left(\frac{\partial S}{\partial H}\right) \frac{d H}{T} \frac{d t}{.}
$$

The modulus in Eq. (5) can be written down as

$$
\begin{aligned}
\left|T_{\mathrm{ac}}\right| & =\frac{P_{\mathrm{ac}}}{\sqrt{\left[\kappa+\left(\frac{\partial S}{\partial H}\right)_{T} \frac{d H}{d t}\right]^{2}+(2 \omega C)^{2}}} \\
& =\frac{P_{\mathrm{ac}}}{2 \omega C}\left\{1+\left[\frac{\left.\left.\kappa+\left(\frac{\partial S}{\partial H}\right)_{T} \frac{d H}{d t}\right]^{2}\right\}^{-1 / 2}=\frac{P_{\mathrm{ac}}}{2 \omega C}|\sin (\varphi)| .}{2 \omega C}\right]\right.
\end{aligned}
$$

For $d H / d t=0$, Eqs. (6) and (7) give the same equations as Ref. 14

$$
\left|T_{\mathrm{ac}}\right|=\frac{P_{\mathrm{ac}}}{2 \omega C}\left[1+\left(\frac{\kappa}{2 \omega C}\right)^{2}\right]^{-1 / 2}=\frac{P_{\mathrm{ac}}}{2 \omega C}|\sin (\phi)|
$$

and

$$
\tan (\phi)=-2 \omega C / \kappa .
$$

Equations (6)-(9) for the ac heat capacity apply in the appropriate measurement frequency range with respect to two time constants. One time constant is determined by the thermal diffusivity $(D)$ and the thickness $(d)$ of the sample, thermometer, and heater ensemble. This is often referred to as the internal time constant $\left(\tau_{2}\right)$, which is associated with the temperature homogeneity inside the sample, thermometer, and heater ensemble. The other time constant, called the external time constant $\left(\tau_{1}\right)$, corresponds to the time required for the system to reach equilibrium with the thermal bath. The proper measurement frequency $(2 f)$ for Eqs. (7) and (8) should be small compared to $1 / \tau_{2}$ and large compared to $1 / \tau_{1}{ }^{27}$ In this frequency range $\left(1 / \tau_{1} \ll 2 f \ll 1 / \tau_{2}\right), \varphi$ is close to $-90^{\circ},\left|T_{\text {ac }}\right|$ is inversely proportional to $C$, and the product of $\left|T_{\text {ac }}\right|$ and frequency becomes constant as a function of $2 f$, where $f$ is defined as $f=\omega / 2 \pi$ [i.e., real part in Eq. (5) is negligible]. Thus, an observation of the plateau in the $\left|T_{\text {ac }}\right| \cdot f$ versus $2 f$ plot is a strong certificate of this frequency condition.

It is quite important to emphasize here that any and all pulsed field experiments are affected by the MCE, i.e., reversibility (the absence of loops) in physical properties measured as a function of magnetic field does not necessarily imply that the sample temperature is constant during the magnetic field pulse, as shown in Fig. 1(c). Our $C_{p}$ measurement under pulsed magnetic fields is no exception, and the MCE term $T(\partial S / \partial H)_{T}(d H / d t)$ might affect $\varphi$ and $T_{\text {ac }}$ in Eqs. (7) and (8), and could induce an error in the evaluated $C$. In addition, the derived equations listed above are stationary solutions, and we need a high enough measurement frequency to achieve approximately an equilibrium condition. Hence, we usually need to check for the effects of MCE to the evaluation of $C$ by changing the magnetic field sweep rate $d H / d t$. This could be a reliable confirmation of the absence of the error caused by MCE. We would note that Eqs. (7) and (8) are derived from the equation of entropy $T d S=\delta q$ for the reversible process. If the system induces heat by an irreversible process such as motion of vortices or magnetic domains, the extra term representing the irreversible process can be added to the initial equation $T d S=\delta q_{\mathrm{re}}+\delta q_{\mathrm{ir}}$. Then, Eq. (2) can be extended as

$$
\begin{aligned}
P & =C \frac{d T}{d t}+T\left(\frac{\partial S}{\partial H}\right)_{T} \frac{d H}{d t}-\left|\frac{\delta q_{\mathrm{ir}}}{d t}\right| \\
& =C \frac{d T}{d t}+\left[T\left(\frac{\partial S}{\partial H}\right)_{T}-\left|\frac{\delta q_{\mathrm{ir}}}{d H}\right|\right] \cdot \frac{d H}{d t}
\end{aligned}
$$

The extra term $\left|\delta q_{\text {ir }} / d H\right|$ represents the amount of heat caused by irreversible processes during field sweep and is always positive (irreversible processes, by definition, never produce cooling). This contribution can impact negatively the determination of $C$ as MCE does, and hence every effort must be made to minimize it. The most common types of irreversible processes are internal dissipative processes at phase transitions and eddy-current heating in metallic materials that always heat the sample disregarding in which direction the magnetic field is changed. While difficult to calculate a priori, the effects of a $\left|\delta q_{\mathrm{ir}} / d H\right|$ term can be estimated with measurements done with different sweep rates, i.e., different $d H / d t$ magnitude and sign. By doing this, the contribution of reversible and irreversible terms is changed inside the brackets in Eq. (10), and the specific heat results are affected. In practical cases, then, when no changes are observed for experiments performed at different $d H / d t$, it is concluded that MCE and irreversible processes are negligible in the determination of $C$. 


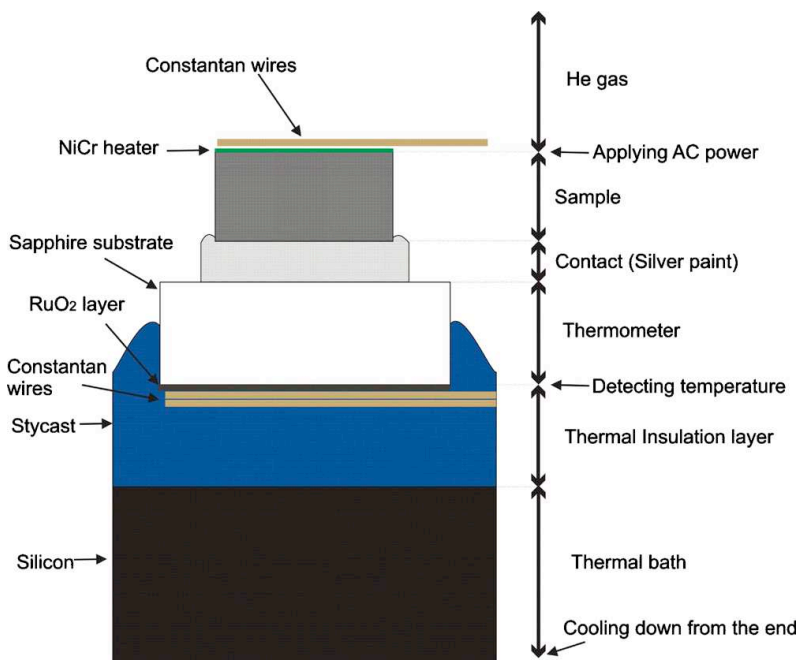

FIG. 2. (Color online) Schematic drawing of our ac calorimeter. The arrows on the right hand side represent the six different regions used in our 1D simple model for simulating the temperature profile in our calorimeter (see Fig. 7.)

\section{EXPERIMENTAL SETUP}

A cross-sectional view of the present calorimeter is sketched in Fig. 2. The calorimeter cell consists of a small bare-chip resistive thermometer and a Si block acting as thermal bath. The small bare-chip resistive thermometer was electrically contacted with Constantan wires of $25.4 \mu \mathrm{m}$ diameter (0.001 in.) using a silver epoxy $\left(\right.$ EPO-TEK $\left.{ }^{\circledR} \mathrm{H} 20 \mathrm{E}\right)$. The face on which electrical contacts were made was directly glued to the Si thermal bath using an epoxy resin $\left(\right.$ Stycast $\left.^{\circledR} 2850\right)$. Extra wires were glued to the Si thermal bath to reduce vibration-related electromagnetic noise. The thickness of the epoxy layer is $\sim 150 \mu \mathrm{m}$, which acts as thermal insulation between the thermometer and the $\mathrm{Si}$ thermal bath. We tune the thermal conductivity between sample and thermal bath with a variable amount of ${ }^{4} \mathrm{He}$ (or ${ }^{3} \mathrm{He}$ ) exchange gas. The small amount of gas makes a small contribution to the heat capacity of the addenda, which we can neglect. Because the thermal conductivity of a gas is determined by the mean translational kinetic energy and the mean free path, ${ }^{28}$ its thermal conductivity is independent of the magnetic field. As resistive thermometers, we tested both Cernox ${ }^{\circledR}$ and $\mathrm{RuO}_{2}$ bare-chips in similar arrangements. The $\mathrm{RuO}_{2}$ resistive thermometer is a commercially available thinfilm chip resistor (State of the Art, Inc., S0302DPG221F-W). This thermometer was polished on one side to a final dimension of $900 \times 500 \times 150 \mu^{3}$. Silver paint (SPI No. 5002) and GE-7031 varnish were used as a thermal contact between sample and thermometer. In the case of ac calorimetry, we used silver paint due to its high thermal conductivity, while for MCE measurements, both contact materials worked well.

In this work, we choose $\mathrm{Sr}_{3} \mathrm{Cr}_{2} \mathrm{O}_{8}, \mathrm{RbFe}\left(\mathrm{MoO}_{4}\right)_{2}$, and $\mathrm{Si}$ single crystals as test samples. The $\mathrm{Sr}_{3} \mathrm{Cr}_{2} \mathrm{O}_{8}$ single crystal samples were grown using an optical floating zone method at McMaster University; ${ }^{29}$ the $\mathrm{RbFe}\left(\mathrm{MoO}_{4}\right)_{2}$ single crystals were grown by a flux method; $;^{30}$ and the $\mathrm{Si}$ single crystals are commercial samples from Insaco, Inc. An $\sim 10 \mathrm{~nm}$ nickelchromium $(\mathrm{NiCr})$ layer was deposited on one side of the sample, to be used as heater. This layer resistance is typically $\sim 10 \mathrm{k} \Omega$ at $4 \mathrm{~K}$ and shows little temperature and magnetic field dependence compared to any thermometer. For example, at $4 \mathrm{~K}$, a $1 \%$ change in the temperature leads to only $0.05 \%$ change in the resistance of the $\mathrm{NiCr}$ layer, which is 20 times smaller than that of the $\mathrm{RuO}_{2}$ thermometer. In our measurement, the magnetoresistance of $\mathrm{NiCr}$ is only $0.1 \%$ up to $45 \mathrm{~T}$, which is 50 times smaller than our $\mathrm{RuO}_{2}$ thermometer. The magnetoresistance and temperature dependence of the thermometers, as well as that of a $\mathrm{NiCr}$ heater, were measured while directly immersed in liquid ${ }^{4} \mathrm{He}$ or high pressure ${ }^{4} \mathrm{He}$ gas in order to maintain high temperature homogeneity between target and reference thermometers. The high thermal conductivity and heat capacity of the sapphire block and the short duration of the magnetic field pulse ensure that the thermometer under calibration remains isothermal while the pulsed magnetic field is applied. For the thermometer calibration, the absence of heating during the field pulse was also confirmed by measuring several resistivity curves with varied magnetic field sweep rates $d H / d t$.

Figures 3(a) and 3(b) show the block diagrams for the data acquisition setup used in the MCE and heat capacity measurement systems, respectively. In the MCE measurement and the calibrations of the thermometers, we used a four contact ac technique to measure thermometer resistance with a digital lock-in system, part of the NHMFL routine measurements of the electric resistance in pulsed magnetic field. ${ }^{31}$ We also measured the magnetoresistance of thermometers with a four contact dc technique, and observed no difference besides the signal to noise ratio between ac technique and dc technique in the down-sweep. A $100 \Omega$ shunt resistor was used to determine the ac current through the $\mathrm{RuO}_{2}$ thermometer. For driving constant ac current, we used a $1 \mathrm{k} \Omega$ resistor and a 1:100 transformer with an in-house ac voltage source. Since the effective impedance in this circuit is quite high $(\sim 10 \mathrm{M} \Omega)$, this method successfully generates a constant ac current during the pulse.

In the heat capacity measurements [Fig. 3(b)], we drive an ac current of frequency $f$ through the $\mathrm{NiCr}$ film heater using an in-house ac voltage source, a resistor, and a transformer, as shown in Fig. 3(b), which induces temperature oscillations of frequency $2 f$ in the sample. The resultant temperature oscillation is detected with a dc technique. To reduce noise, we choose a commercial battery equipped with an $\sim 400 \mathrm{k} \Omega$ resistor as a current source for the dc detection. In a separate experiment we confirmed that the change in the current during the field pulse is negligibly small. For some experiments a Keithley 220 current source was also used. The amplitude and the phase of ac current flowing in the heater were measured by means of a $100 \Omega$ resistance. The oscillating signal in the thermometer was split and amplified via two Stanford Research SR560 preamplifiers, where one preamplifier measures the entire signal without filtering the $\mathrm{dc}$ component and another preamplifier filtered the dc component. After amplification by factors of 10-100, the signals were stored in our data acquisition computer by using commercial National Instruments ${ }^{\circledR}$ and $\mathrm{GaGe}$ Applied 

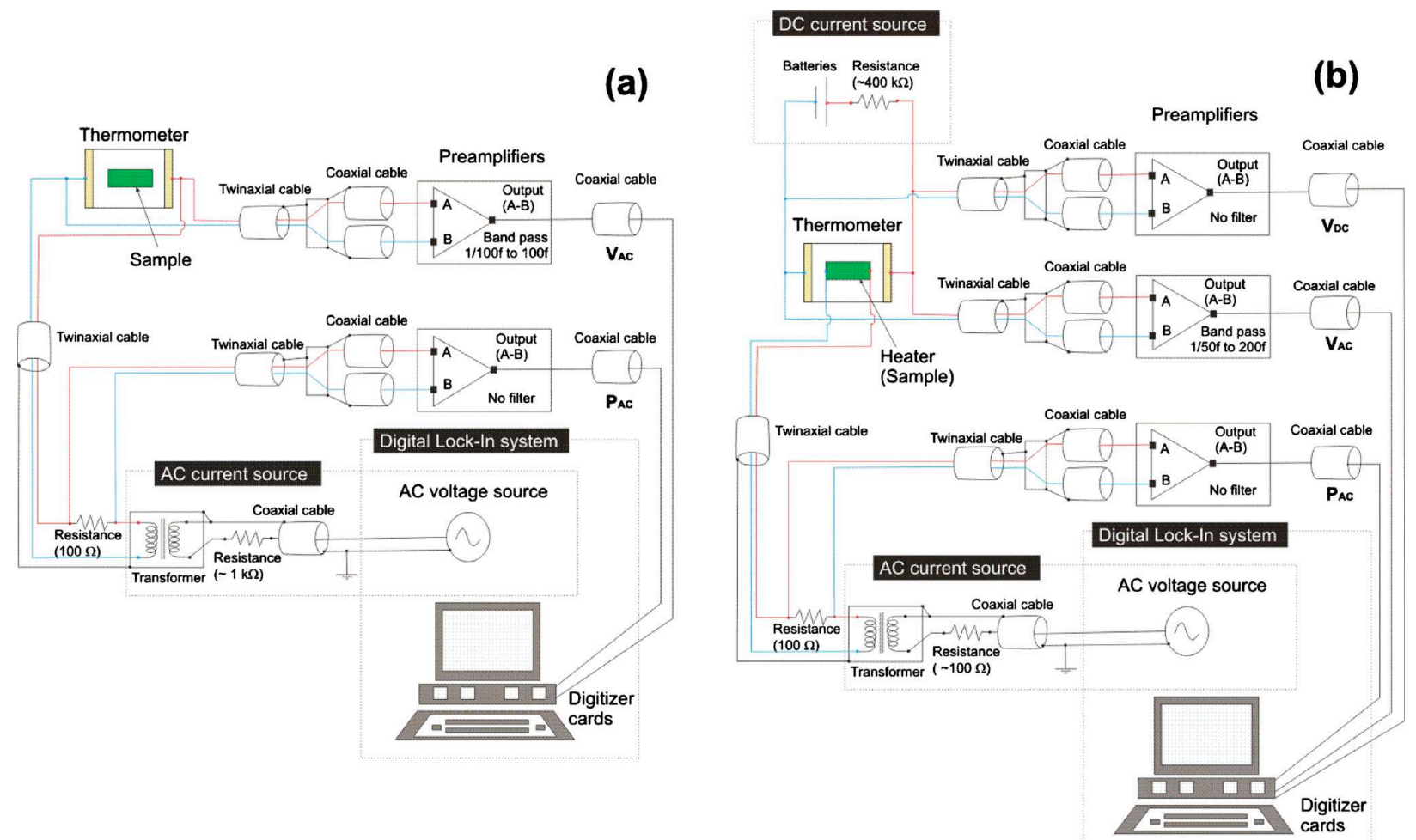

FIG. 3. (Color online) Circuit diagrams for (a) MCE and (b) heat capacity measurements. (a) The ports labeled $\mathrm{V}_{\mathrm{ac}}$ and $\mathrm{P}_{\mathrm{ac}}$ detect the resistance and the ac current changes in the thermometer, respectively. The preamplifier at the $\mathrm{V}_{\mathrm{ac}}$ port removes the dc component and noise with the band-pass filter. The band-pass filter blocks signals with a frequency 100 times higher or lower than the ac current frequency $(f)$. (b) $V_{d c}, V_{a c}$, and $P_{a c}$ are the ports to detect the resistance change of thermometer, the amplified ac resistance oscillation of the thermometer, and the applied ac power to the NiCr heater during experiments, respectively. The band-pass filter at the $\mathrm{P}_{\mathrm{ac}}$ port rejects the signal with frequencies far from the ac temperature oscillation frequency $(2 f)$.

Technologies ${ }^{\circledR}$ digitizers. In both measurements, the digitizers collected the time dependence of the signal using a data acquisition rate of $0.1-20 \mathrm{M}$ samples/s.

While we have collected and reported ${ }^{26} \mathrm{MCE}$ data taken in a $250 \mathrm{~ms}$ and $2 \mathrm{~s}$ pulsed magnet, for clarity we limit our discussion here to measurements in a $50 \mathrm{~T}$ capacitor-bank driven pulsed magnet with $250 \mathrm{~ms}$ pulse duration. During the magnetic field up-sweep, the average rate is $50 \mathrm{~T} / 25 \mathrm{~ms}$ $=2000 \mathrm{~T} / \mathrm{s}$, while the magnetic field during the down-sweep changes much more slowly. The down-sweep rates, although time-dependent, are approximately 300 and $150 \mathrm{~T} / \mathrm{s}$ when peak fields are 40 and $15 \mathrm{~T}$, respectively.

\section{MAGNETORESISTANCE OF CERNOX ${ }^{\circledR}$ AND RuO $_{2}$ THERMOMETERS}

When performing heat capacity and MCE measurements in high magnetic fields at low temperature, it is quite important to correctly account for the magnetoresistance of the resistive thermometer. However, most reports on resistive thermometers have been limited to the magnetic field range accessible to dc magnets. ${ }^{32,33}$ In this section, we briefly report the magnetoresistance of $\mathrm{RuO}_{2}$ and Cernox thermometers measured in a pulsed magnet and describe an advantage of $\mathrm{RuO}_{2}$ thermometers for heat capacity and MCE experiments.

Figures 4(a) and 4(b) show three-dimensional (3D) plots of magnetoresistance up to $\sim 6 \mathrm{~K}$ for a Cernox ${ }^{\circledR}$ thermom- eter (CX-1010) and a $\mathrm{RuO}_{2}$ thermometer. Both thermometers show clear magnetoresistance with decreasing temperature. The CX-1010 thermometer exhibits complex (both positive and negative) magnetoresistance below $10 \mathrm{~K}$. For example, at about $3 \mathrm{~K}$, the magnetoresistance shows a small bump at low fields. Similar magnetoresistance has been reported previously. ${ }^{32,33}$ On the other hand, the $\mathrm{RuO}_{2}$ thermometer shows monotonic positive magnetoresistance, and its relative change $\left[R\left(\begin{array}{ll}45 & \mathrm{~T}\end{array}\right) / R\left(\begin{array}{ll}0 \mathrm{~T}\end{array}\right)\right]$ only varies from $5 \%$ to $6 \%$ in the measurement temperature range. Additionally, while the $\mathrm{RuO}_{2}$ sensor gains temperature sensitivity $(d R / d T$ becomes larger) at high fields, the CX1010 loses significant $d R / d T$ at similar fields. These features favor the $\mathrm{RuO}_{2}$ resistance thermometer for use in high magnetic fields. On the other hand, while Cernox ${ }^{\circledR}$ thermometers behave nicely at high frequencies, $\mathrm{RuO}_{2}$ thermometers show somewhat strong frequency dependence in its resistance and thus we typically choose relatively low frequencies $(1-2 \mathrm{kHz})$ for our measurements. The observed frequency dependence $(1 \%$ at $5 \mathrm{kHz})$ might be an intrinsic feature of $\mathrm{RuO}_{2}$ resulting from the dielectric glass matrix. ${ }^{34}$ When using frequencies above $\sim 3 \mathrm{kHz}$, it is then necessary to recalibrate the magnetoresistance of $\mathrm{RuO}_{2}$ at each frequency.

The relatively small and monotonic magnetoresistance in the $\mathrm{RuO}_{2}$ thermometer allows us to fit the magnetoresistance with the simple polynomial function, 

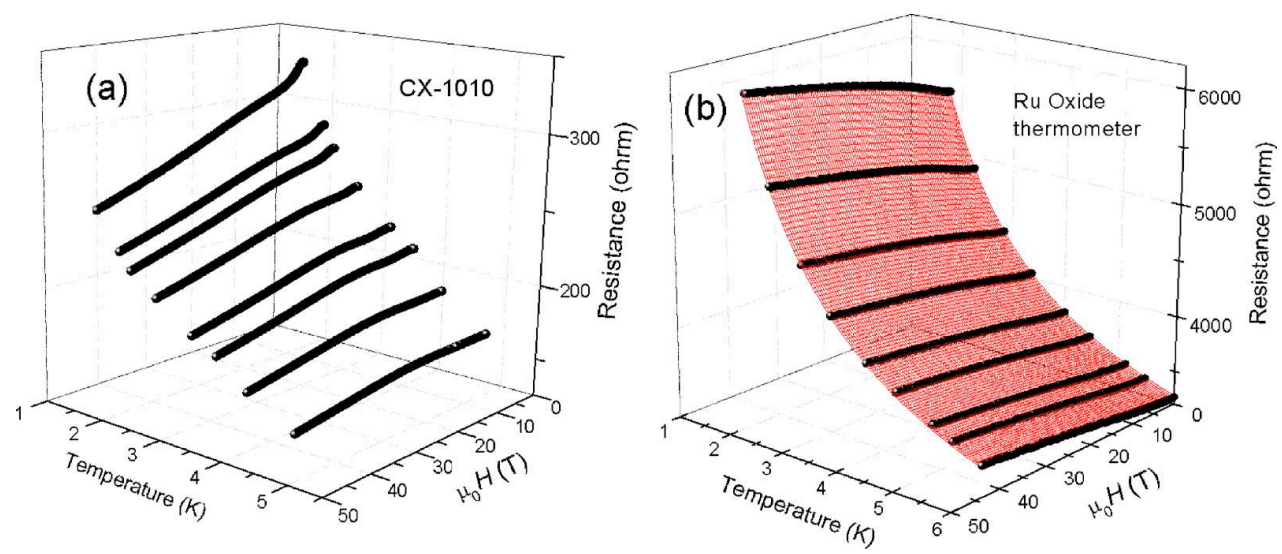

FIG. 4. (Color online) 3D plot of the magnetoresistance of the (a) CX-1010 and (b) $\mathrm{RuO}_{2}$ thermometers at low temperatures in fields up to 50 T. The resistances of the CX-1010 and the $\mathrm{RuO}_{2}$ thermometers were measured at 60 and $2 \mathrm{kHz}$, respectively. The red surface plot is the result of the surface fitting using the polynomial function discussed in the text.

$$
\begin{aligned}
R_{\mathrm{fit}}(H, T)= & a_{0}+a_{1} H^{0.5}+a_{2} T^{-1}+a_{3} H+a_{4} T^{-2} \\
& +a_{5} H^{0.5} T^{-1}+a_{6} H^{1.5}+a_{7} T^{-3} \\
& +a_{8} H^{0.5} T^{-2}+a_{9} H T^{-1},
\end{aligned}
$$

where $a_{n}$ for $n=0,1, \ldots, 9$ are the polynomial coefficients. As shown in Fig. 4(b) with a red surface, the fitting function has an excellent agreement with data. The deviation of the fitting function from the data points is within $\sim 0.2 \%$ over the entire range of temperatures and magnetic fields. This error corresponds to $\sim 20, \sim 10$, and $\sim 4 \mathrm{mK}$ at 6,4 , and 2 $\mathrm{K}$, respectively.

\section{MAGNETOCALORIC EFFECT RESULTS}

To test advantages and shortcomings of MCE and heat capacity measurements in pulsed magnet field, we used a single crystal sample of the spin dimmer compound $\mathrm{Sr}_{3} \mathrm{Cr}_{2} \mathrm{O}_{8}$ which shows XY-type antiferromagnetism between $H_{c 1}=30.4 \mathrm{~T}$ and $H_{c 2}=62 \mathrm{~T}$. The $\left(H_{c}, T_{c}\right)$ phase boundary for this quantum paramagnet system has a dome shape that is limited to $T_{\text {cmax }} \sim 8 \mathrm{~K}$ in temperature, ${ }^{26}$ qualitatively similar to other systems previously studied. ${ }^{5,11,13}$ This family of compounds has a nonmagnetic spin-singlet ground state, with a finite energy gap to the first excited spin-triplet states. A strong enough external magnetic field can be used to close the spin gap, inducing magnetic ordering. Hence, because of the closing of the gap and the induced magnetic ordering, $\Delta S$ shows a peak structure as a function of magnetic field which is discussed in the work by Aczel et $a .^{26}$ The MCE results expected in this system include a crossover-type behavior of the type depicted in Fig. 1(d), but with the opposite sign because $S$ increases with field in our sample and also a phase transition as in Fig. 1(e). This behavior has been observed before in $\mathrm{Ba}_{3} \mathrm{Mn}_{2} \mathrm{O}_{8}$ at lower fields produced with dc resistive magnets.

We show MCE data for $\mathrm{Sr}_{3} \mathrm{Cr}_{2} \mathrm{O}_{8}$ taken at $T=2.4$ and 4.5 $\mathrm{K}$ in Fig. 5(a). Here, the sample dimensions are 300 $\times 350 \times 100 \mu^{3}$. The up-sweep data show a broader dip, while the down-sweep data show a sharp peak. At $2.4 \mathrm{~K}$, the magnetocaloric response is not sharp probably due to the smaller entropy change at the phase boundary. In our data, the MCE curve clearly consists of two typical magnetocaloric responses described by Figs. 1(d) and 1(e) superimposed. Below $\sim 30 \mathrm{~T}$, the magnetocaloric response corre-
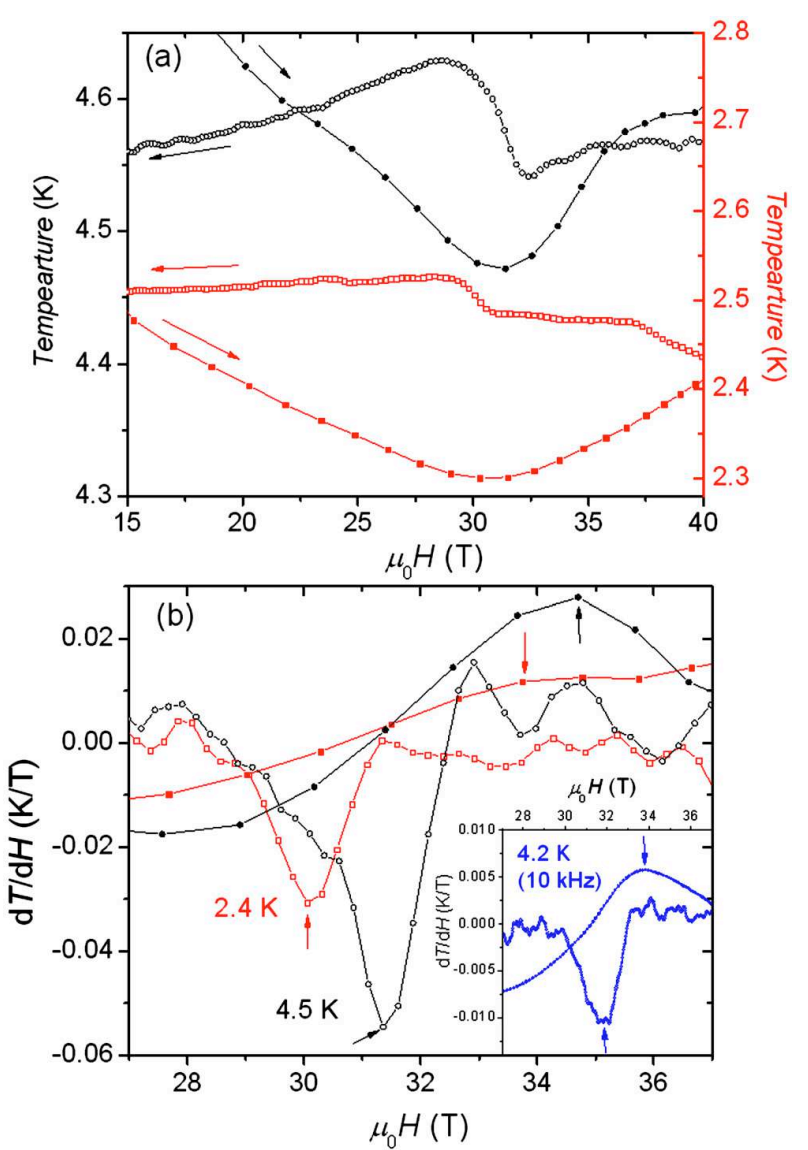

FIG. 5. (Color online) (a) MCE data for $\mathrm{Sr}_{3} \mathrm{Cr}_{2} \mathrm{O}_{8}$. The solid and open symbols are data taken during the up-sweep and down-sweep, respectively. The circles and squares are data measured at 4.5 and $2.4 \mathrm{~K}$, respectively. (b) Numerical derivative of the MCE data. The peak in the up-sweep at $2.4 \mathrm{~K}$ is not obvious due to the slowly varying MCE response. The data in the inset were taken at $4.2 \mathrm{~K}$ using a measurement frequency of $10 \mathrm{kHz}$, while the data in the main panel were taken at $1 \mathrm{kHz}$. The arrows indicate the peak positions. 
sponds to the entropy increase due to the closing of the spin gap. Above $\sim 30 \mathrm{~T}$, a careful inspection of the up-sweep and down-sweep data taken at $4.5 \mathrm{~K}$ reveals a sharper temperature change, which is related to the second order magnetic phase transition. Although a quantitative evaluation of $S$ is difficult, the symmetric response indicates that a reversible process is predominant in this magnetic phase transition. In fact, the MCE measurement in dc field shows a clear, symmetric MCE at $H_{\mathrm{c} 1} \cdot{ }^{26}$ The evaluation of $H_{\mathrm{c} 1}$ from pulsed field data can be done by taking the first derivative of temperature versus field $\partial T / \partial H$, as shown in Fig. 5(b). One peak is observed for each sweep and the peak position in the upsweep is higher than in the down-sweep. The difference between up- and down-sweeps can be explained by the sample temperature lagging behind during the fast up-sweep, which was also observed in the experiments using dc magnets with high sweep rates. This is because the sample temperature needs a finite time characterized by $\tau_{1}$ to respond to the heat released from the entropy change, as in traditional thermal relaxation type calorimetry. Since the delay should be proportional to the sweep rate $(d H / d t)$, the critical magnetic field $H_{\mathrm{c}}$ can be evaluated from the following equation:

$$
\begin{aligned}
H_{c}= & \left(H_{c, \text { peak-down }} \frac{d H}{d t}\right. \text { up } \\
& \left.+H_{c \text {, peak-up }} \frac{d H}{d t} \text { down }\right) /\left(\frac{d H}{d t} \text { up }+\frac{d H}{d t} \text { down }\right) .
\end{aligned}
$$

The resulting $H_{\mathrm{cl}}(T)$ is $30.8 \mathrm{~T}$ at $2.4 \mathrm{~K}$ and $32.1 \mathrm{~T}$ at $4.5 \mathrm{~K}$, with the departure from the phase diagram derived from data collected in dc fields is less than $1 \mathrm{~T}^{26}$ We believe that the main source of error is the delay in the sample response $\left(\tau_{1}\right.$ is not small enough) and the small number of data points. The use of a smaller sample and a higher measurement frequency can reduce the error. As an example, the derivative data at 4.2 $\mathrm{K}$ measured at $10 \mathrm{kHz}$ with a smaller sample (200 $\times 300 \times 50 \mu^{3}$ ) are plotted in the inset of Fig. 5(b). The clean data obtained this way show less separation between the up-sweep and down-sweep peaks, which allows us to obtain the phase boundary more precisely.

\section{FREQUENCY TEST AND NUMERICAL SIMULATIONS}

In MCE measurements, a delay of the thermal response leads to broad MCE curves and makes it difficult to evaluate the correct field-temperature phase diagram in magnetic materials. The time scale of the delay could be the "external" time constant $\left(\tau_{1}\right)$ as described in the previous chapter, which is typically a few orders of magnitude larger than the "internal" time constant $\left(\tau_{2}\right)$. If we apply the ac calorimetric technique, we can dramatically reduce the time scale of the delay from $\tau_{1}$ to $\tau_{2}$ and construct a more precise phase diagram. In addition, the evaluation of $C(H)$ gives us a chance to discuss the implications of the physical pictures.

In Fig. 6, we plot experimental results of $\left|T_{\text {ac }}\right| \cdot f$ versus $2 f$ and $\varphi$ for $\mathrm{Sr}_{3} \mathrm{Cr}_{2} \mathrm{O}_{8}$ and $\mathrm{Si}$ single crystals, which were measured at $\sim 4 \mathrm{~K}$ and zero magnetic field. In this frequency test, the sample dimensions for the $\mathrm{Sr}_{3} \mathrm{Cr}_{2} \mathrm{O}_{8}$ and $\mathrm{Si}$ single crystals are $450 \times 250 \times 100$ and $450 \times 200 \times 130 \mu^{3}$, which have heat capacities of $1.1 \times 10^{-8}$ and 5.4 $\times 10^{-10} \mathrm{~J} \mathrm{~K}^{-1}$, respectively. The $\mathrm{Sr}_{3} \mathrm{Cr}_{2} \mathrm{O}_{8}$ sample shows a clear plateau in $\left|T_{\mathrm{ac}}\right| \cdot f$ between 1 and $10 \mathrm{kHz}$, while the $\mathrm{Si}$ sample only exhibits two kinks due to its small contribution to the total heat capacity. The phase $\varphi$ is close to $-90^{\circ}$ near $2 \mathrm{kHz}$ for both samples. In the $\mathrm{Sr}_{3} \mathrm{Cr}_{2} \mathrm{O}_{8}$ sample, the frequency dependences observed in $\left|T_{\mathrm{ac}}\right| \cdot f$ and $\varphi$ indicate that the frequency range near $2 \mathrm{kHz}$ for $2 f\left(\sim 6283 \mathrm{~s}^{-1}\right.$ for $\left.\omega\right)$ closely satisfies the requirements $\left(1 / \tau_{1} \ll 2 f \ll 1 / \tau_{2}\right)$ for the ac measurement of $C_{p}$. In the case of the $\mathrm{Si}$ sample, we should keep in mind that the addenda contribution to the total heat capacity can decrease with increasing measurement frequency, even if the frequency condition $\left(1 / \tau_{1} \ll 2 f\right.$ $\left.\ll 1 / \tau_{2}\right)$ is fulfilled, because the variation of the thermal length with measurement frequency can affect the addenda heat capacity. This addenda heat capacity originates from the He exchange gas, heater, thermometer, silver paint, electrical wires, and Stycast. Therefore, the small heat capacity of Si in comparison with the addenda heat capacity might explain the lack of a plateau in the Si frequency test.

In order to understand the specific features of our ac calorimeter and the frequency test results in $\mathrm{Si}$, we solved a simple one-dimensional heat equation model using a finite element method, which is commonly used for finding approximate solutions for the heat equation. ${ }^{35}$ The homemade computation program was built in a LABVIEW ${ }^{\mathrm{TM}} 8.2$ package from National Instruments. The vertical arrows on the right hand side of Fig. 2 represent the construction of our model, where the model consists of six regions, ${ }^{4} \mathrm{He}$ gas, sample, contact, thermometer, thermal insulation layer, and Si thermal bath. For simplicity, we neglected the thin $\mathrm{RuO}_{2}$ layer, $\mathrm{NiCr}$ layer, and the Constantan wires. We chose a $30 \mu \mathrm{m}$ thickness and $0.1 \mathrm{~W} \mathrm{~K}^{-1} \mathrm{~m}^{-1}$ thermal conductivity for the contact layer (silver paint). ${ }^{36}$ Other physical values used in this calculation, such as heat capacity and thermal conductivity in each region, are taken from the literature $;^{36-48}$ these values are listed in the figure caption of Fig. 7. Figure 7(a) shows the simulated temperature profile within these six regions at $2 f=1 \mathrm{kHz}\left(\omega \sim 3142 \mathrm{~s}^{-1}\right)$. The thermal oscillations caused by the ac power transfer from the top surface of the sample to the end of the thermometer equipped with a thin $\mathrm{RuO}_{2}$ layer, although the contact layer reduces by $10 \%$ the thermal oscillation amplitude at this frequency. In the region of the thermal insulation layer (Stycast), the temperature oscillation is damped and then the high thermal conductance $\mathrm{Si}$ thermal bath does not show any thermal oscillations. Since we observe the heat capacity in the region where the temperature is oscillating, this result confirms that our calorimeter measures the heat capacity of the sample, contact layer, thermometer, and a layer of ${ }^{4} \mathrm{He}$ gas and thermal insulation layer. The heat capacity in ${ }^{4} \mathrm{He}$ gas $\left(173.9 \mathrm{~J} \mathrm{~K}^{-1} \mathrm{~m}^{-3}\right.$ at 2.5 mbar $){ }^{48}$ silver paint $\left(2400 \mathrm{~J} \mathrm{~K}^{-1} \mathrm{~m}^{-3}\right),{ }^{45}$ and Stycast $\left(1400 \mathrm{~J}^{-1} \mathrm{~K}^{-1} \mathrm{~m}^{-3}\right.$ ) (Ref. 44) cannot be neglected, when we measure a small specific heat sample such as $\mathrm{Sr}_{3} \mathrm{Cr}_{2} \mathrm{O}_{8}$ $\left(1000 \mathrm{~J}^{-1} \mathrm{~K}^{-1} \mathrm{~m}^{-3}\right.$ ) (Ref. 26) and especially $\mathrm{Si}$ $\left(45.7 \mathrm{~J}^{-1} \mathrm{~K}^{-1} \mathrm{~m}^{-3}\right) .{ }^{46}$ However, since we can write down the measured heat capacity $\left(C^{\mathrm{tot}}\right)$ as $C^{\mathrm{tot}}=C^{\text {addenda }}+C^{\mathrm{Sr}_{3} \mathrm{Cr}_{2} \mathrm{O}_{8}}$ or $C^{\text {addenda }}+C^{\mathrm{Si}}$, the addenda heat capacity can be eliminated by the following equation: 


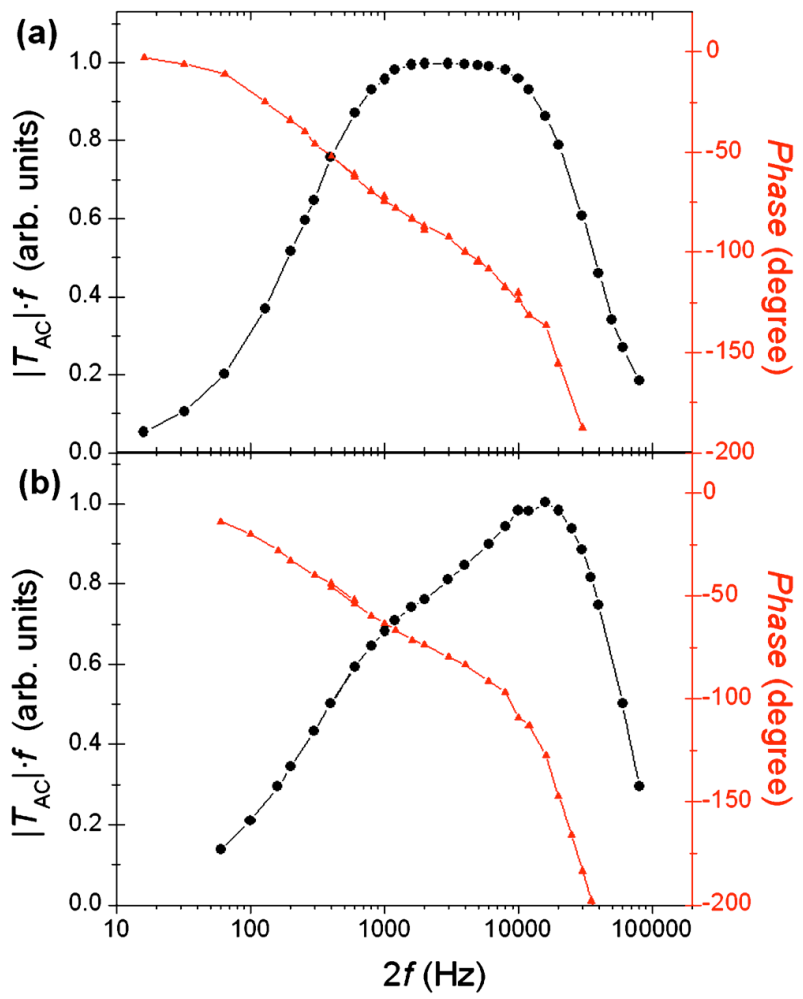

FIG. 6. (Color online) Frequency dependence of $\left|T_{\mathrm{ac}}\right| \cdot 2 f$ and $\varphi$ for $\mathrm{Sr}_{3} \mathrm{Cr}_{2} \mathrm{O}_{8}$ (a) and $\mathrm{Si}$ (b) single crystals. $\left|T_{\mathrm{ac}}\right| \cdot 2 f$ and $\varphi$ are represented by circles and triangles, respectively. The maximum value of $\left|T_{\mathrm{ac}}\right| \cdot 2 f$ at the top of the dome is normalized to 1 .

$$
\frac{P_{\mathrm{ac}}^{\mathrm{Sr}_{3} \mathrm{Cr}_{2} \mathrm{O}_{8}}}{2 \omega \cdot T_{\mathrm{ac}}^{\mathrm{Sr}_{3} \mathrm{Cr}_{2} \mathrm{O}_{8}}}-\frac{P_{\mathrm{ac}}^{\mathrm{Si}}}{2 \omega \cdot T_{\mathrm{ac}}^{\mathrm{Si}}} \approx C^{\mathrm{Sr}_{3} \mathrm{Cr}_{2} \mathrm{O}_{8}}-C^{\mathrm{Si}} .
$$

The variables on the left-hand side are observables and we can determine $C^{\mathrm{Sr}_{3} \mathrm{Cr}_{2} \mathrm{O}_{8}}-C^{\mathrm{Si}}$. Since $C^{\mathrm{Si}}$ is negligible small and also already reported elsewhere ${ }^{46}$ we successfully evaluated the $C$ of the sample in pulsed magnetic fields.

Figures 7(b) and 7(c) are the calculated $\left|T_{\text {ac }}\right| \cdot f$ versus $2 f$ curves for $\mathrm{Sr}_{3} \mathrm{Cr}_{2} \mathrm{O}_{8}$ and $\mathrm{Si}$ samples. The shapes of the curves in $\left|T_{\mathrm{ac}}\right| \cdot f$ and $\varphi$ are similar to the experimental result in Figs. 6(a) and 6(b). The simulated $\left|T_{\mathrm{ac}}\right| \cdot f$ curve for the $\mathrm{Sr}_{3} \mathrm{Cr}_{2} \mathrm{O}_{8}$ sample shows a slightly rounded plateau between 1 and $10 \mathrm{kHz}$. The clear shape of the plateau in the experiment seems to stem from the contribution of the extra silver paint to the total heat capacity because the larger heat capacity can increase $\tau_{1}$ and extend the size of the plateau. The phase difference $\varphi$ in $\mathrm{Sr}_{3} \mathrm{Cr}_{2} \mathrm{O}_{8}$ is close to $-90^{\circ}$ near $2 \mathrm{kHz}$, similar to the simulation result in Fig. 6, which is the desired condition in ac calorimetry. On the other hand, the $\left|T_{\mathrm{ac}}\right| \cdot f$ in the Si sample shows only kinks at $500 \mathrm{~Hz}$ and $10 \mathrm{kHz}$ and $\varphi$ is close to $-90^{\circ}$ near $4 \mathrm{kHz}$. The absence of plateau could be caused by the change of the addenda heat capacity with frequency because the contribution of the Si to total heat capacity is much smaller than the heat capacity from the addenda. As shown in Fig. 7(b), the heat capacity change in the sample can be detected as a decrease of the $\left|T_{\mathrm{ac}}\right|$, but the thermal conductivity change from 1 to $25 \mathrm{~W} / \mathrm{K}^{-1} \mathrm{~m}^{-1}$ does
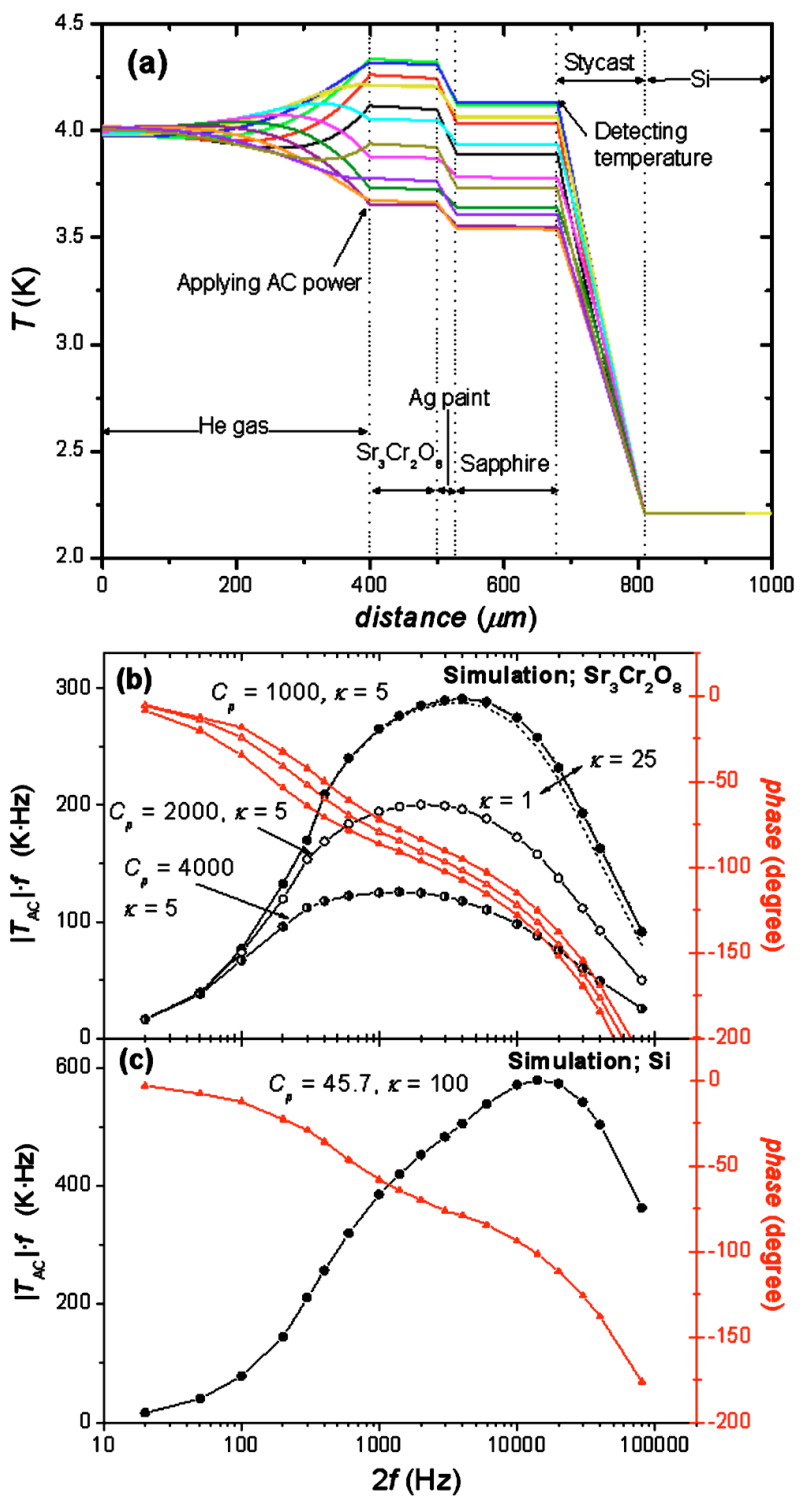

FIG. 7. (Color online) (a) Simulated temperature profile in our calorimeter at various times. The 12 curves have $6 / \pi$ phase intervals for the ac heating power. In this calculation, we used specific heats of 45.7 (Si), 2400 (silver paint), $8.63\left(\alpha \mathrm{Al}_{2} \mathrm{O}_{3}\right), 1400$ (Stycast), $1000-4000 \quad\left(\mathrm{Sr}_{3} \mathrm{Cr}_{2} \mathrm{O}_{8}\right)$ and $173.9 \mathrm{~J} \mathrm{~K}^{-1} \mathrm{~m}^{-3}\left({ }^{4} \mathrm{He}\right.$ at $\left.2.5 \mathrm{mbar}\right)$ and thermal conductivities of $100(\mathrm{Si})$, 0.1 (silver paint), $100\left(\alpha \mathrm{Al}_{2} \mathrm{O}_{3}\right), 0.04$ (Stycast), 1-25 $\left(\mathrm{Sr}_{3} \mathrm{Cr}_{2} \mathrm{O}_{8}\right)$ and $0.00767 \mathrm{~W} \mathrm{~K}^{-1} \mathrm{~m}^{-1}\left({ }^{4} \mathrm{He}\right)$. The applied ac power $\left(P_{\mathrm{ac}}\right)$ is $5 \times 10^{-10} \mathrm{~W}$ over a $1 \mu \mathrm{m}^{2}$ area and the thermal conductance from the edge of the silicon platform is $5 \times 10^{-6} \mathrm{~W} \mathrm{~K}^{-1}$. The time step and distance step are 50-5000 ns and $1 \mu \mathrm{m}$. (b) Simulated frequency dependence of $\left|T_{\mathrm{ac}}\right| \cdot f$ and $\varphi$ in $\mathrm{Sr}_{3} \mathrm{Cr}_{2} \mathrm{O}_{8}$ single crystal. The solid, open, and half-solid circles are the $\left|T_{\mathrm{ac}}\right| \cdot f$ with $C=1000$ and $\kappa=5, C=2000$ and $\kappa=5$, and $C=4000$ and $\kappa=5$. The black dashed and dot curves are the results for $C=1000$ and $\kappa=1$ and $C=1000$ and $\kappa=25$, respectively. The solid, open, and half-solid triangles are $\varphi$ with $C=1000$ and $\kappa=5, C=2000$ and $\kappa=5$, and $C=4000$ and $\kappa=5$. (c) Simulated frequency dependence of $\left|T_{\text {ac }}\right| \cdot f$ and $\varphi$ for a Si single crystal.

not affect $\left|T_{\mathrm{ac}}\right|$. This suggests that our setup can measure sample heat capacity, but not its thermal conductivity.

Moreover, we can conclude that the damping in $\left|T_{\mathrm{ac}}\right|$ happens in a low thermal diffusivity layer that is likely the thermal contact layer in our calorimeter. Even if some temperature damping exists, we still can measure heat capacity 
by evaluating how the $\left|T_{\mathrm{ac}}\right|$ is reduced in the contact layer. In the low frequency limit $\left(f \ll \kappa / 4 \pi C L^{2}\right)$, where $L$ is the thickness of a layer, Ivanda and Djurek ${ }^{49}$ gave an expression for the temperature oscillations of a low thermal diffusivity layer sandwiched between a heater and another low thermal diffusivity layer,

$$
T(x) \approx\left[A_{1}+A_{2} \exp (i \omega t)\right] \frac{\kappa_{T}|x|+\kappa_{C} L_{T}}{\kappa_{T} L_{C}+\kappa_{C} L_{T}},
$$

where $A_{1}$ and $A_{2}$ are constants, $\kappa_{T}, L_{T}$ and $\kappa_{C}, L_{C}$ are the thermal conductivity and the thickness of each low thermal diffusivity layer. By neglecting the temperature gradients in the sample and thermometer layers, which commonly have higher thermal diffusivities than that of the contact layer, the equation approximates the temperature oscillation of the thermal contact layer in our ac calorimeter. In this case, $\kappa_{T}$, $L_{T}$ and $\kappa_{C}, L_{C}$ correspond to the thermal conductivity and the thickness of the thermal insulation and contact layers in our calorimeter, respectively. By taking the ratio of amplitudes at two different positions $L_{C}$ and 0 on the contact layer, we can get

$$
\frac{\left|T_{\mathrm{ac}}(0)\right|}{\left|T_{\mathrm{ac}}\left(L_{C}\right)\right|} \approx \frac{\kappa_{C} L_{T}}{\kappa_{T} L_{C}+\kappa_{C} L_{T}} .
$$

Then, Eq. (8) can be rewritten as $\left|T_{\mathrm{ac}}\right| \sim-a P_{\mathrm{ac}} / 2 \omega C \sin \varphi$, where $a$ is equal to the right hand side of Eq. (15). Since $\kappa_{T} L_{C}$ in Eq. (15) is more than ten times smaller than $\kappa_{C} L_{T}$ in our ac calorimeter $\left(\kappa_{T} \sim 0.04 \mathrm{~W} \mathrm{~K}^{-1} \mathrm{~m}^{-1},{ }^{38} L_{\mathrm{T}} \sim 150 \mu \mathrm{m}\right.$, $\kappa_{C} \geq 0.1 \mathrm{~W} \mathrm{~K}^{-1} \mathrm{~m}^{-1},{ }^{36}$ and $\left.L_{C} \sim 30 \mu \mathrm{m}\right), a$ is close to 1 and expected not to vary dramatically with changing $\kappa_{T}$ and $\kappa_{C}$ in magnetic fields. For example, if we assume a $50 \%$ decrease in $\kappa_{C}$ upon application of external magnetic fields, likely an overestimate for the polycrystalline silver paint, the expected change in $a$ is only $7 \%$. Thus, we can neglect the change in $a$ with magnetic field.

\section{HEAT CAPACITY RESULTS}

Figure 8(a) shows the overall temperature change during a magnetic field pulse recorded for a heat capacity measurement with a Si single crystal. The black and gray curves are data taken during the up- and down-sweeps, respectively, and the light gray curve is smoothed data. The $2 f$ temperature oscillations of $1 \mathrm{kHz}\left(\sim 3142 \mathrm{~s}^{-1}\right.$ for $\left.\omega\right)$ are clearly observed in both the up- and down-sweep data (also seen in the enlarged inset figure). Because of the lower $d H / d t$, the downsweep curve shows a large number of temperature oscillations than the up-sweep curve. The heating effect due to the high $d H / d t$ in the up-sweep results in a small temperature increase at about $5 \mathrm{~T}$. Except for the low field region of the up-sweep, almost no heating effect is detected, which indicates that the MCE is negligibly small.

By multiplying the sinusoidal reference functions of frequency $2 f$ by the observed signal, as is usually done in a commercial digital lock-in amplifier, we numerically extracted the $\left|T_{\mathrm{ac}}\right|$ component after integration over one period without any further smoothing process. Figure $8(\mathrm{~b})$ provides the measured $P_{\mathrm{ac}} / 2 \omega \cdot\left|T_{\mathrm{ac}}\right|$ in the Si sample at $\sim 4.2 \mathrm{~K}$ in a pulsed field up to $43 \mathrm{~T}$, which correspond to $C^{\text {addenda }}+C^{\mathrm{Si}}$.

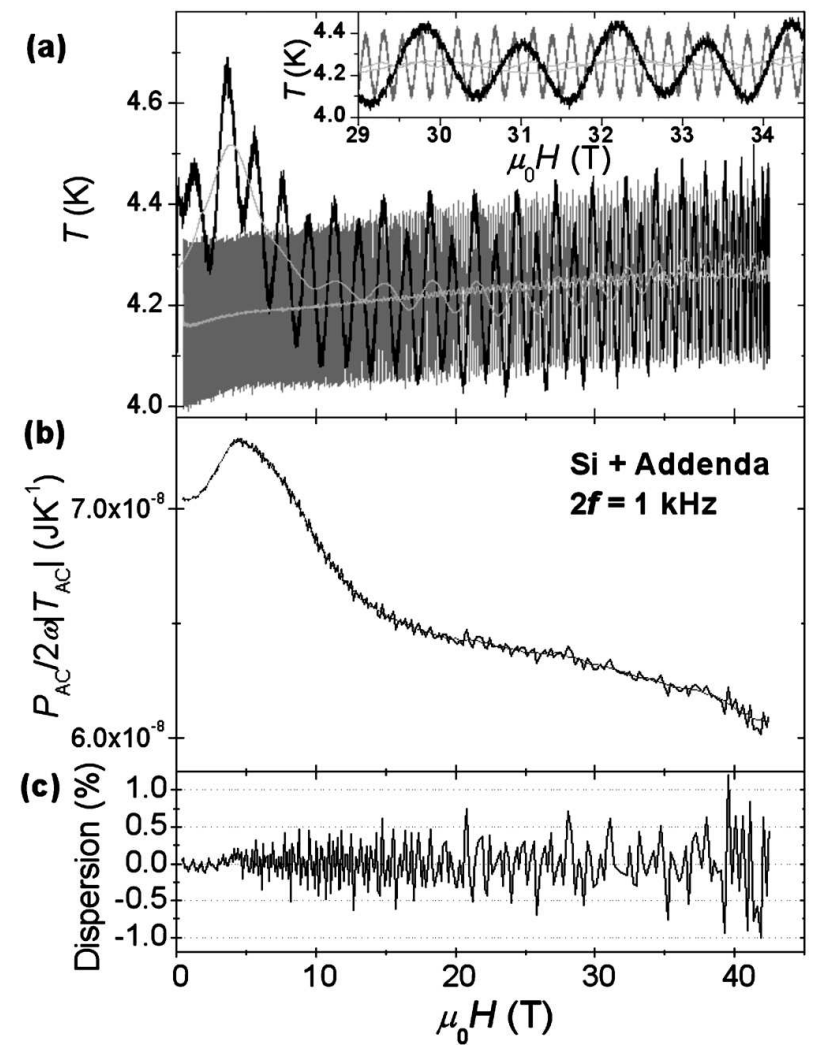

FIG. 8. (a) Temperature vs magnetic field for the Si single crystal during the $250 \mathrm{~ms}$ pulse. The frequency of the temperature oscillation $(2 f)$ is $1 \mathrm{kHz}$ $\left(\omega \sim 3142 \mathrm{~s}^{-1}\right)$. The signal was detected without filtering the raw signal. (b) Magnetic field dependence of $P_{\mathrm{ac}}^{\mathrm{Si}} / 2 \omega \cdot\left|T_{\mathrm{ac}}^{\mathrm{Si}}\right|$. For the purpose of clarity, we do not show the $P_{\mathrm{ac}}^{\mathrm{Si}} / 2 \omega \cdot\left|T_{\mathrm{ac}}^{\mathrm{Si}}\right|$ data extracted from the data of up-sweep, which do not have a high enough measurement frequency for the quickly rising magnetic field and show noise at about $5 \mathrm{~T}$ due to the small temperature increase as shown in Fig. 8(a). (c) The deviation of the data points from the smoothed curve corresponding to the sensitivity of this measurement.

The black curve is evaluated from the raw temperature data of the downward sweeping magnetic field, and the light gray curve is its smoothed curve. In these curves, one broad bump at low magnetic field can be observed. The size of the bump is only $\sim 10 \mathrm{~nJ} \mathrm{~K}{ }^{-1}$ at $\sim 5 \mathrm{~T}$ and its broad shape reminds us of a Schottky anomaly. In fact, the measurement temperature of $\sim 4 \mathrm{~K}$ corresponds to the energy gap of free spins caused by Zeeman splitting. ${ }^{50}$ Therefore, a tiny amount of free impurity spins contained in $\mathrm{Si}$ and/or calorimeter seems to be responsible for this broad anomaly. Above $20 \mathrm{~T}$, the data are almost independent of the magnetic field. In this field region, the magnitude of the heat capacity is two orders of magnitude higher than the expected heat capacity of $\mathrm{Si}$ $\left(5.4 \times 10^{-10} \mathrm{~J} \mathrm{~K}^{-1}\right)$. This confirms that the addenda heat capacity is the major contribution in this measurement. Indeed, specific heats of Stycast ${ }^{44}$ and silver paint ${ }^{45}$ are two orders of magnitude larger than that of $\mathrm{Si}^{46}$ Hence, we can use the measured $P_{\mathrm{ac}} / 2 \omega \cdot\left|T_{\mathrm{ac}}\right|$ in this $\mathrm{Si}$ measurement as the heat capacity of the addenda and can evaluate the sample heat capacity with Eq. (13). Figure 8(c) displays the deviation of the data points from the smoothed curve as a function of the magnetic field. With increasing the magnetic field, the dispersion of data becomes slightly larger due to the electronic 
noise, but it is still within $\pm \sim 0.5 \%$. At higher frequency, the dispersion is increased due to the smaller $\left|T_{\mathrm{ac}}\right|$. For example, without smoothing, the dispersions at $43 \mathrm{~T}$ are $\pm 1 \%, \pm 5 \%$, and $\pm 15 \%$ at $2 f=2,5$, and $10 \mathrm{kHz}$, respectively. With a large number of data points in the high frequency region, smoothing can effectively reduce the dispersion of the data points.

In order to confirm that our calorimeter has the sensitivity to detect heat capacity anomalies as a function of temperature and magnetic field, we measured the triangular lattice antiferromagnet system $\mathrm{RbFe}\left(\mathrm{MoO}_{4}\right)_{2}$. In zero field, this sample shows quite a large heat capacity anomaly $\left[3.8 \times 10^{5} \mathrm{~J} \mathrm{~K}^{-1} \mathrm{~m}^{-3}\right.$ at $\sim 3.74 \mathrm{~K}$ (Ref. 51) and 5.7 $\times 10^{5} \mathrm{~J} \mathrm{~K}^{-1} \mathrm{~m}^{-3}$ at $3.90 \mathrm{~K}$ (Ref. 52)], and by applying external magnetic fields, several magnetic ordered states are induced. ${ }^{51-53}$ Since the detected signal $\left|T_{\text {ac }}\right|$ is inversely proportional to the heat capacity, the large heat capacity in $\mathrm{RbFe}\left(\mathrm{MoO}_{4}\right)_{2}$ is quite difficult to measure. The sizes of samples mounted on our calorimeter are $130 \times 250$ $\times 50 \mu \mathrm{m}^{3}$ (sample 1) and $120 \times 330 \times 70 \mu \mathrm{m}^{3}$ (sample 2) which have much larger heat capacities [(6.2-15.9) $\times 10^{-7} \mathrm{~J} \mathrm{~K}^{-1}$ at peak temperature] than the addenda heat capacity shown in Fig. 8.

The squares and triangles in the inset of Fig. 9(a) are $\left|T_{\text {ac }}\right| \cdot f$ versus $2 f$ in samples 1 and 2 , respectively. Both samples show peaks at around $100 \mathrm{~Hz}$, which are quite different from those in $\mathrm{Sr}_{3} \mathrm{Cr}_{2} \mathrm{O}_{8}$ and $\mathrm{Si}$ single crystals. The origin of the difference can be explained by the large specific heat of $\mathrm{RbFe}\left(\mathrm{MoO}_{4}\right)_{2}$, resulting in large $\tau_{1}$ and $\tau_{2}$. These large time constants shift the plateau to low frequencies and are able to change the shape of plateau. Although the absence of a plateau makes it difficult to evaluate the absolute value of the heat capacity, we tested the ac measurements of heat capacity at $2 f=400 \mathrm{~Hz} \quad\left(\omega \sim 1257 \mathrm{~s}^{-1}\right)$, which is the lowest frequency limit of our setup. Figure 9(a) depicts the

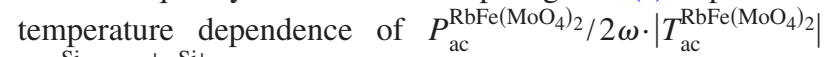
$-P_{\mathrm{ac}}^{\mathrm{Si}} / 2 \omega \cdot\left|T_{\mathrm{ac}}^{\mathrm{Si}}\right|$, which was taken with a temperature ramp rate of $2.5 \mathrm{~K} \mathrm{~s}^{-1}$ at $4 \mathrm{~K}$. The black, blue, and red curves were measured with ac powers of 5.8, 8.3, and $16 \mu \mathrm{W}$, respectively. The data taken by the traditional relaxation method $^{52}$ (green circles) are on the right axis. Strictly speak-

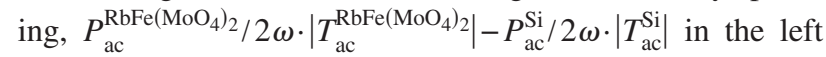
axis does not correspond to $C^{\mathrm{RbFe}\left(\mathrm{MoO}_{4}\right)_{2}}-C^{\mathrm{Si}}$, because no plateau behavior in $\left|T_{\text {ac }}\right| \cdot f$ versus $2 f$ makes difficult to apply Eqs. (8) and (13) in this measurement. In fact, the evaluated heat capacity of $\mathrm{RbFe}\left(\mathrm{MoO}_{4}\right)_{2}$ becomes six to nine times larger than the heat capacity values measured using the traditional thermal relaxation time technique. This is because the absence of a plateau reduces $\left|T_{\mathrm{ac}}^{\mathrm{RbFe}\left(\mathrm{MoO}_{4}\right)_{2}}\right|$, and

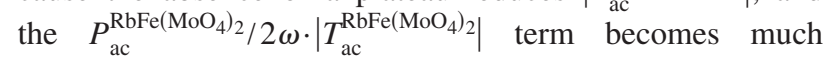
larger than the true heat capacity. However,

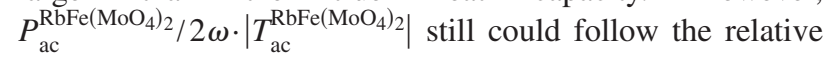
changes of the heat capacity. Indeed, the three curves measured by our ac calorimeter agree with the $C(T)$ data in the Ref. 52. The agreement between data sets tells us that the main advantage of our calorimeter is its high measurement frequency and sensitivity. These allow us to complete quick measurements during fast temperature and magnetic field sweeps. In fact, each curve in Fig. 9(a) was taken within
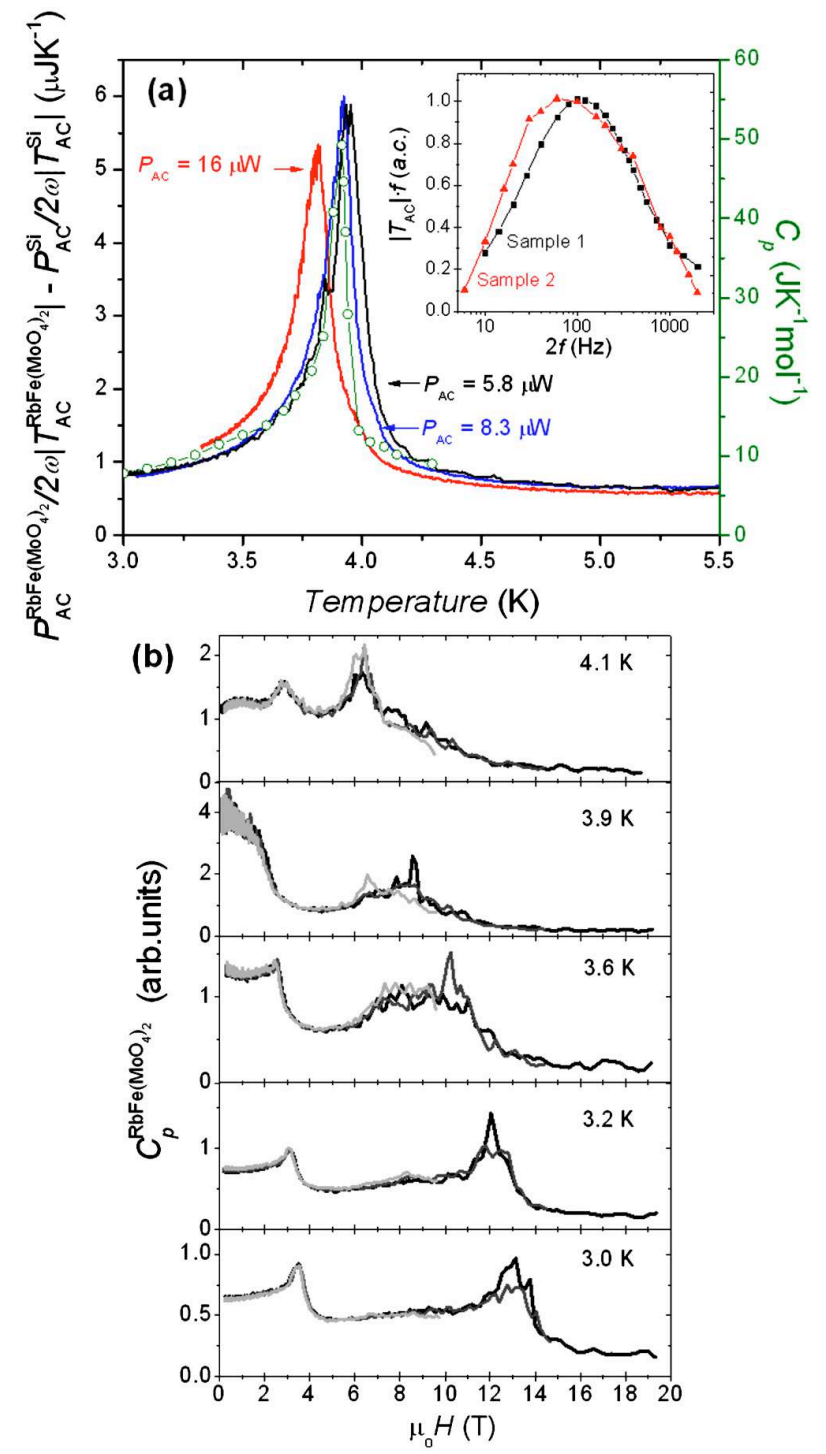

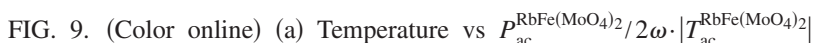
$-P_{\mathrm{ac}}^{\mathrm{Si}} / 2 \omega \cdot\left|T_{\mathrm{ac}}^{\mathrm{Si}}\right|$ for sample 1, a $\mathrm{RbFe}\left(\mathrm{MoO}_{4}\right)_{2}$ single crystal. The frequency of the temperature oscillation $(2 f)$ is $400 \mathrm{~Hz}\left(\omega \sim 1257 \mathrm{~s}^{-1}\right)$. The inset shows $\left|T_{\mathrm{ac}}\right| \cdot f$ vs $2 f$ plot for samples 1 (squares) and 2 (triangles). (b) $C^{\mathrm{RbFe}\left(\mathrm{MoO}_{4}\right)_{2}}$ in arbitrary units up to $20 \mathrm{~T}$ (sample 1 ). Using the temperature oscillation data taken during down-sweeps, these $C^{\mathrm{RbFe}\left(\mathrm{MoO}_{4}\right)_{2}}$ data were cal-

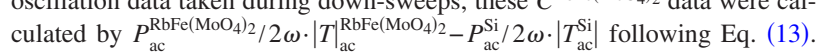
The black, gray, and light gray curves were measured under 20,15 , and 10 $\mathrm{T}$ pulsed magnetic fields, respectively.

$\sim 1$ s. A limitation of ac power is expected in the cases of sample and/or contact, showing very large heat capacity and/or bad thermal conductivity. Actually, the peak temperatures in the three curves $(3.81,3.92$, and $3.94 \mathrm{~K}$ at $16,8.3$, and $5.8 \mu \mathrm{W}$, respectively) are shifted to lower temperature when a higher ac power is applied to the heater. This is due to the existence of a temperature gradient between the thermometer and the sample. This temperature gradient can also be found in the numerical results in Fig. 7(a), where the application of $5 \times 10^{-10} \mathrm{~W} / \mu \mathrm{m}^{2}$ grid (corresponding to $16.25 \mu \mathrm{W}$ in sample 1) gives an average temperature difference of $\sim 0.15 \mathrm{~K}$ at the silver paint contact layer. Thus, a 
small ac power must be applied to avoid large temperature gradients. In the following measurements of $\mathrm{RbFe}\left(\mathrm{MoO}_{4}\right)_{2}$ in pulsed fields, we choose $8.3 \mu \mathrm{W}$ for $P_{\text {ac }}$.

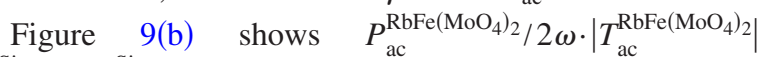
$-P_{\mathrm{ac}}^{\mathrm{Si}} / 2 \omega \cdot\left|T_{\mathrm{ac}}^{\mathrm{Si}}\right|$ versus magnetic field up to $20 \mathrm{~T}$. Here, the black, gray, and light gray curves are measured with maximum magnetic fields of 20,15 , and $10 \mathrm{~T}$, which have magnetic field sweep rates $d H / d t$ of 47,35 , and $23 \mathrm{~T} / \mathrm{s}$

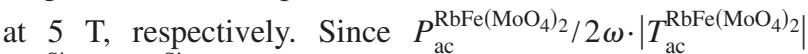
$-P_{\mathrm{ac}}^{\mathrm{Si}} / 2 \omega \cdot\left|T_{\mathrm{ac}}^{\mathrm{Si}}\right|$ seem to be roughly proportional to the heat capacity in Fig. 9(a), we labeled it as $C^{\mathrm{RbFe}\left(\mathrm{MoO}_{4}\right)_{2}}$ with arbitrary units. In the whole measurement temperature and magnetic fields range, $C^{\mathrm{RbFe}\left(\mathrm{MoO}_{4}\right)_{2}}$ shows pronounced anomalies. All of the observed anomalies in this measurement nicely reproduce previous heat capacity data taken using the traditional relaxation method ${ }^{53}$ and follow the known phase diagram. ${ }^{51-53}$ Here, we want to point out that the three curves taken with changing $d H / d t$ show the same shape of the peaks especially in the high sensitivity low field region. This means the change of $(\partial S / \partial H)_{T}(d H / d t)$ in Eqs. (6) and (7) is negligibly small, and we could use Eq. (8) in these pulsed field measurements. The low sensitivity at high magnetic fields especially near peak positions is caused by the electric noise in pulsed fields, which is not negligible compared to the small, detected $\left|T_{\mathrm{ac}}\right|$ of $\sim 2 \mathrm{mK}$ in this measurement. However, typical samples have a much smaller heat capacity than $\mathrm{RbFe}\left(\mathrm{MoO}_{4}\right)_{2}$ and should show larger $\left|T_{\mathrm{ac}}\right|$ and higher sensitivity. The heat capacity data in $\mathrm{RbFe}\left(\mathrm{MoO}_{4}\right)_{2}$ as a function of temperature and magnetic field indicate that our calorimeter efficiently maps out the field-temperature phase diagram for magnetic materials.

Figures 10(a) and 10(b) show the temperature oscillations at $2 f=1 \mathrm{kHz}\left(\omega \sim 3142 \mathrm{~s}^{-1}\right)$ during a heat capacity measurement of a $\mathrm{Sr}_{3} \mathrm{Cr}_{2} \mathrm{O}_{8}$ single crystal. The black and gray curves taken during up- and down-sweeps show the ac temperature oscillations. The amplified signal in Fig. 10(b) and its inset clearly detect the ac temperature oscillations with a high sensitivity of $\pm 2 \mathrm{mK}$ at $\sim 30 \mathrm{~T}$. The averaged raw signal (gray curve) in Fig. 10(a) exhibits the MCE at 33.1 and 32.1 T in the up- and down-sweeps. These magnetic fields are consistent with MCE data in pulsed magnetic field (see Fig. 5), and the transition magnetic field determined by Eq. (12) agrees with the previous heat capacity and MCE data in dc field. ${ }^{26}$ The agreement observed in the determination of critical magnetic field indicates that the temperature gradient between sample and thermometer caused by the ac power is negligibly small in this measurement. By numerically extracting $\left|T_{\mathrm{ac}}\right|$ from the temperature oscillation

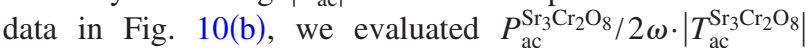
$-P_{\text {ac }}^{\mathrm{Si}} / 2 \omega \cdot\left|T_{\mathrm{ac}}^{\mathrm{Si}}\right|$, which is simply refereed to as $C^{\mathrm{Sr}_{3} \mathrm{Cr}_{2} \mathrm{O}_{8}}$ in Fig. 10(c). These data show a pronounced peak and a small dip at 32.7 and $32.1 \mathrm{~T}$, respectively. The peak shape agrees with the heat capacity data measured in dc magnetic fields (green circles) ${ }^{26}$ which again confirms the reliability of our ac calorimeter. The dips indicated by the arrows, however, seem to be artificial signals caused by the MCE. One possible explanation of dips is the $(\partial S / \partial H)_{T}(d H / d t)$ term in Eq. (7), which could affect $\left|T_{\mathrm{ac}}\right|$ and $C$. Another explanation is

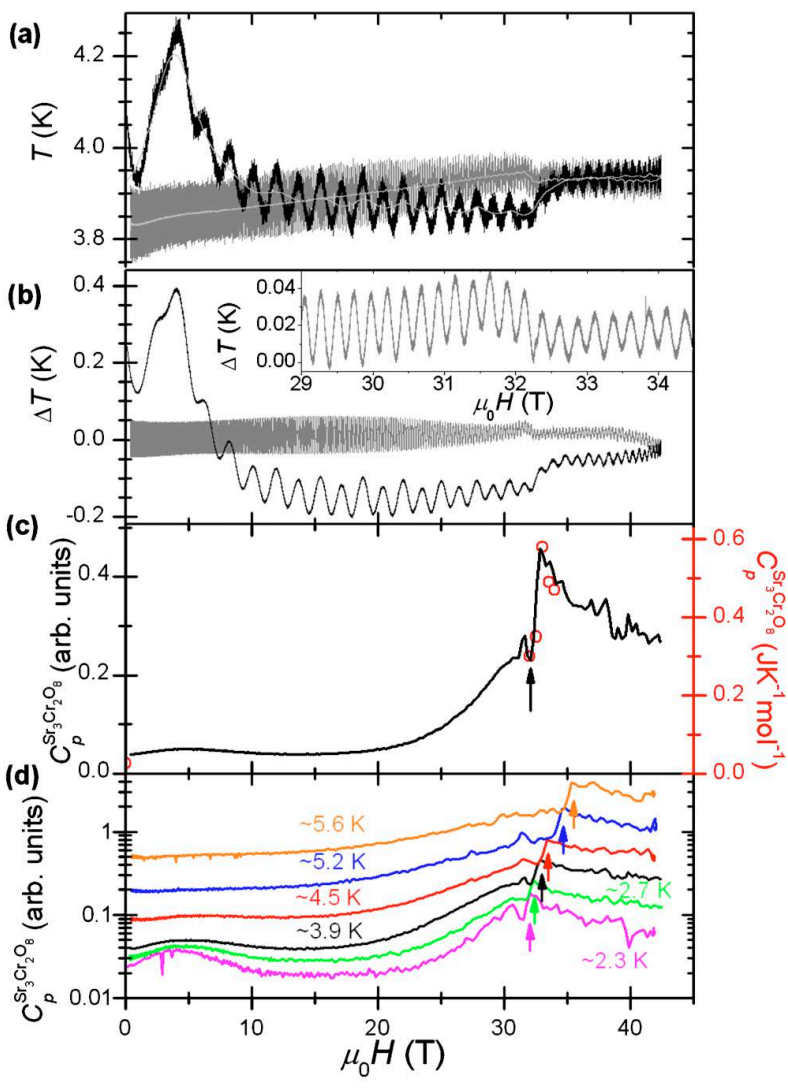

FIG. 10. (Color online) (a) Temperature vs magnetic field during the $250 \mathrm{~ms}$ pulse for a $\mathrm{Sr}_{3} \mathrm{Cr}_{2} \mathrm{O}_{8}$ single crystal. The frequency of temperature oscillation $(2 f)$ is $1 \mathrm{kHz}\left(\omega \sim 3142 \mathrm{~s}^{-1}\right)$. The gray, black, and light gray curves are the data from the down-sweep, up-sweep, and smoothed data. (b) Temperature oscillation vs magnetic field. These temperature oscillation data were detected after filtering and amplifying the raw resistance oscillation signal with a preamplifier [see the electronic circuit diagram in Fig. 3(b)]. The inset is the enlarged figure in the field range of the spin-ordering transition of $\mathrm{Sr}_{3} \mathrm{Cr}_{2} \mathrm{O}_{8}$. (c) The black curve is $C^{\mathrm{Sr}_{3} \mathrm{Cr}_{2} \mathrm{O}_{8}}$ in arbitrary units, which corre-

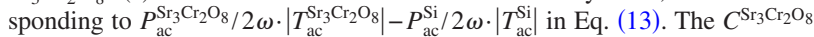
data were calculated from the temperature oscillation data taken during down-sweeps. The open circles are the heat capacity data measured in dc field (Ref. 26) and the arrows indicate the dips observed at the field showing MCE. (d) $C^{\mathrm{Sr}_{3} \mathrm{Cr}_{2} \mathrm{O}_{8}}$ between 2.3 and $5.6 \mathrm{~K}$. The arrows indicate the peaks in the $C_{p}$ data.

that errors in the numerical evaluation process of $\left|T_{\mathrm{ac}}\right|$ might occur when the raw temperature signal shows a sudden jump. In fact, the size of the jumps seen in Fig. 10(b) and its inset are compatible with the $\left|T_{\mathrm{ac}}\right|$ which might bring an artificial noise. In any case, the size of this dip is negligible when compared with the big peak at the spin-ordering phase transition. Figure 10(d) displays the $C^{\mathrm{Sr}_{3} \mathrm{Cr}_{2} \mathrm{O}_{8}}$ measured from 2.3 to $5.6 \mathrm{~K}$. The peaks at the spin-ordering phase transition are clearly observed in all temperature regions and the temperature dependence again agrees with the previous heat capacity and MCE measurement. ${ }^{26}$ The larger dip evident at lower temperatures could be caused by a larger MCE than reported for experiments performed in dc fields ${ }^{26}$ and the broad peak at $5 \mathrm{~T}$ should correspond to a Schottky anomaly caused by probable impurity spins in the $\mathrm{Sr}_{3} \mathrm{Cr}_{2} \mathrm{O}_{8}$ sample. The consistencies of the peak magnetic fields with the previous report are satisfactory and demonstrate that our calorimeter works reliably in a $250 \mathrm{~ms}$ pulsed magnetic field. 
Finally, we would like to mention a few issues and future plans to improve our calorimeter. The first issue of our heat capacity measurement is the evaluation of the contribution of the silver paint to the total heat capacity. This is originated from the difficulty to determine the amounts of silver paint used for the thermal contact to the thermometer and the electrical contact to the $\mathrm{NiCr}$ heater, which represents a nonnegligible contribution to the total heat capacity. A second issue is that a deposited $\mathrm{NiCr}$ heater does not work on metallic samples due to the high conductivity of the sample itself. To solve these problems, the application of the optical heating technique should be most effective and it could increase the sensitivity of our calorimeter. These tests are currently under way.

\section{ACKNOWLEDGMENTS}

Y.K. and M.J. would like to thank D. F. Weickert and Y. Suzuki for early discussions of the ac calorimetry for pulsed field, F. F. Balakirev for the help with digital lock-in measurements, and J. B. Betts and A. Migliori for technical support during the experiment. We are indebted to A. A. Aczel, G. Luke, A. Ya. Shapiro, and L. A. Demianets for providing the $\mathrm{Sr}_{3} \mathrm{Cr}_{2} \mathrm{O}_{8}$ and $\mathrm{RbFe}\left(\mathrm{MoO}_{4}\right)_{2}$ single crystals used for these proof-of-principle experiments. We gratefully acknowledge the help of S. A. Baily and D. Braithwaite during the revision process. This work was supported by the National Science Foundation, the U.S. Department of Energy, and the State of Florida.

${ }^{1}$ M. Jaime, R. Movshovich, G. R. Stewart, W. P. Beyermann, M. G. Berisso, M. F. Hundley, P. C. Canfield, and J. L. Sarrao, Nature (London) 405, 160 (2000)

${ }^{2}$ F. Bouquet, C. Marcenat, E. Steep, R. Calemczuk, W. K. Kwok, U. Welp, G. W. Crebtree, R. A. Fisher, N. E. Philips, and A. Schilling, Nature (London) 411, 448 (2001).

${ }^{3}$ M. Jaime, K. H. Kim, G. Jorge, S. McCall, and J. A. Mydosh, Phys. Rev. Lett. 89, 287201 (2002).

${ }^{4}$ C. R. Rotundu, H. Tsujii, Y. Takano, B. Andraka, H. Sugawara, Y. Aoki, and H. Sato, Phys. Rev. Lett. 92, 037203 (2004).

${ }^{5}$ M. Jaime, V. F. Correa, N. Harrison, C. D. Batista, N. Kawashima, Y. Kazuma, G. A. Jorge, R. Stern, I. Heinmaa, S. A. Zvyagin, Y. Sasago, and K. Uchinokura, Phys. Rev. Lett. 93, 087203 (2004).

${ }^{6}$ J. S. Kim, N. O. Moreno, J. L. Sarrao, J. D. Thompson, and G. R. Stewart, Phys. Rev. B 69, 024402 (2004)

${ }^{7}$ H. Tsujii, Z. Honda, B. Andraka, K. Katsumata, and Y. Takano, Phys. Rev. B 71, 014426 (2005).

${ }^{8}$ T. Plackowski, Y. Wang, R. Lortz, A. Junod, and Th. Wolf, J. Phys.: Condens. Matter 17, 6871 (2005).

${ }^{9}$ A. V. Silhanek, M. Jaime, N. Harrison, V. R. Fanelli, C. D. Batista, H. Amitsuka, S. Nakatsuji, L. Balicas, K. H. Kim, Z. Fisk, J. L. Sarrao, L. Civale, and J. A. Mydosh, Phys. Rev. Lett. 96, 136403 (2006).

${ }^{10}$ R. Lortz, Y. Wang, A. Demuer, P. H. M. Böttger, B. Bergk, G. Zwicknagl, Y. Nakazawa, and J. Wosnitza, Phys. Rev. Lett. 99, 187002 (2007).

${ }^{11}$ E. C. Samulon, Y.-J. Jo, P. Sengupta, C. D. Batista, M. Jaime, L. Balicas, and I. R. Fisher, Phys. Rev. B 77, 214441 (2008).

${ }^{12}$ E. C. Samulon, Y. Kohama, R. D. McDonald, M. C. Shapiro, K. A. Al-Hassanieh, C. D. Batista, M. Jaime, and I. R. Fisher, Phys. Rev. Lett. 103, 047202 (2009)

${ }^{13}$ A. A. Aczel, Y. Kohama, M. Jaime, K. Ninos, H. B. Chan, L. Balicas, H. A. Babkowska, and G. M. Luke, Phys. Rev. B 79, 100409 (2009).

${ }^{14}$ P. F. Sullivan and G. Seidel, Phys. Rev. 173, 679 (1968).

${ }^{15}$ M. Chung, Y. Wang, J. W. Brill, X.-D. Xiang, R. Mostovoy, J. G. Hou, and A. Zettl, Phys. Rev. B 45, 13831 (1992).

${ }^{16}$ C. Marcenat, S. Blanchard, J. Marcus, L. M. Paulius, C. J. van der Beek, M. Konczykowski, and T. Klein, Phys. Rev. Lett. 90, 037004 (2003).
${ }^{17}$ V. A. Bondarenko, S. Uji, T. Terashima, C. Terakura, S. Tanaka, S. Maki, J. Yamada, and S. Nakatsuji, Synth. Met. 120, 1039 (2001).

${ }^{18}$ O. Bourgeois, S. E. Skipetrov, F. Ong, and J. Chaussy, Phys. Rev. Lett. 94, 057007 (2005).

${ }^{19}$ R. Geer, C. C. Huang, R. Pindak, and J. W. Goodby, Phys. Rev. Lett. 63, 540 (1989).

${ }^{20}$ C. W. Garland, Thermochim. Acta 88, 127 (1985).

${ }^{21}$ S. Imaizumi, K. Suzuki, and I. Hatta, Rev. Sci. Instrum. 54, 1180 (1983).

${ }^{22}$ J.-L. Garden, H. Guillou, A. F. Lopeandia, J. Richard, J.-S. Heron, G. M. Souche, F. R. Ong, B. Vianay, and O. Bourgeois, Thermochim. Acta 492, 16 (2009)

${ }^{23}$ A. Demuer, C. Marcenat, J. Thomasson, R. Calemczuk, B. Salce, P. Lejay, D. Braithwaite, and J. Flouquet, J. Low Temp. Phys. 120, 245 (2000).

${ }^{24}$ T. Park, F. Ronning, H. Q. Yuan, M. B. Salamon, R. Movshovich, J. L. Sarrao, and J. D. Thompson, Nature (London) 440, 65 (2006).

${ }^{25}$ R. Lortz, A. Junod, D. Jaccard, Y. Wang, C. Meingast, T. Masui, and S. Tajima, J. Phys.: Condens. Matter 17, 4135 (2005).

${ }^{26}$ A. A. Aczel, Y. Kohama, C. Marcenat, F. Weickert, O. E. AyalaValenzuela, M. Jaime, R. D. McDonald, S. D. Selesnic, H. A. Dabkowska, and G. M. Luke, Phys. Rev. Lett. 103, 207203 (2009).

${ }^{27}$ G. M. Schmiedeshoff, N. A. Fortune, J. S. Brooks, and G. R. Stewart, Rev. Sci. Instrum. 58, 1743 (1987).

${ }^{28}$ S. Blundell and K. Blundell, Concepts in Thermal Physics (Oxford University Press, New York, 2006), Chap. 9.

${ }^{29}$ A. A. Aczel, H. A. Dabkowska, P. R. Provencher, and G. M. Luke, J. Cryst. Growth 310, 870 (2008).

${ }^{30}$ R. F. Klevtosova and P. V. Klevtsov, Kristallografiya 15, 209 (1970).

${ }^{31}$ Y. Kohama, Y. Kamihara, S. A. Baily, L. Civale, S. C. Riggs, F. F. Balakirev, T. Atake, M. Jaime, M. Hirano, and H. Hosono, Phys. Rev. B 79, 144527 (2009)

${ }^{32}$ B. L. Brandt, D. W. Liu, and L. G. Rubin, Rev. Sci. Instrum. 70, 104 (1999).

${ }^{33}$ B. Zhang, J. S. Brooks, J. A. A. J. Perenboom, S.-Y. Han, and J. S. Qualls, Rev. Sci. Instrum. 70, 2026 (1999).

${ }^{34}$ M. Süßer and F. Wüchner, Cryogenics 40, 413 (2000).

${ }^{35}$ R. W. Lewis, K. Morgan, H. R. Thomas, and K. N. Seetharamu, The Finite Element Method in Heat Transfer Analysis (Wiley, New York, 1996).

${ }^{36}$ The cutoff frequency in $\left|T_{\text {ac }}\right| \cdot f$ vs $2 f$ becomes approximately five times higher by changing contact material from GE varnish to silver paint. This suggests that the thermal conductivity of the silver paint is roughly approximately five times higher than that of GE varnish. Since the thermal conductivity of GE varnish is about $0.05 \mathrm{~W} \mathrm{~K}^{-1} \mathrm{~m}^{-1}$ (Ref. 37), we estimate the lower bound for the thermal conductivity of the silver paint to be $0.1 \mathrm{~W} \mathrm{~K}^{-1} \mathrm{~m}^{-1}$

${ }^{37}$ J. H. McTaggart and G. A. Slack, Cryogenics 9, 384 (1969).

${ }^{38}$ F. Rondeaux, Ph. Bredy, and J. M. Rey, Adv. Cryog. Eng. 614, 197 (2002).

${ }^{39}$ C. J. Glassbrenner and G. A. Slack, Phys. Rev. 134, A1058 (1964).

${ }^{40}$ R. Berman, E. L. Foster, and J. M. Ziman, Proc. R. Soc. London 231, 130 (1955).

${ }^{41}$ A. Acton and K. Kellner, Physica B 90, 192 (1977).

${ }^{42} \mathrm{Sr}_{3} \mathrm{Cr}_{2} \mathrm{O}_{8}$ is one of several quantum magnets which has a relatively high thermal conductivity at low temperature; $\mathrm{NaV}_{2} \mathrm{O}_{5}, \kappa=\sim 10 \mathrm{~W} \mathrm{~K}^{-1} \mathrm{~m}^{-1}$ at $5 \mathrm{~K}$ (Ref. 43), $\mathrm{Cu}_{2} \mathrm{Te}_{2} \mathrm{O}_{5} \mathrm{Cl}_{2}, \kappa=\sim 10 \mathrm{~W} \mathrm{~K}^{-1} \mathrm{~m}^{-1}$ at $8 \mathrm{~K}$ (Ref. 43), and $\mathrm{SrCu}_{2}\left(\mathrm{BO}_{3}\right)_{2}, \kappa=\sim 13 \mathrm{~W} \mathrm{~K}^{-1} \mathrm{~m}^{-1}$ at $4 \mathrm{~K}$ (Ref. 43). Thus, we choose relatively high $\kappa$ in the range of $1-25 \mathrm{~W} \mathrm{~K}^{-1} \mathrm{~m}^{-1}$ for $\mathrm{Sr}_{3} \mathrm{Cr}_{2} \mathrm{O}_{8}$ and simulated the ideal $\left|T_{\mathrm{ac}}\right|$ dependence as a function of $2 f$. As shown in Fig. 7(b), a broad plateau is observed up to $10 \mathrm{kHz}$ with low $\kappa$ of $1 \mathrm{~W} \mathrm{~K}^{-1} \mathrm{~m}^{-1}$, which means that our setup can be used with a relatively small $\kappa$ sample.

${ }^{43}$ A. Smontara, A. Bilučić, Z. Japličić, A. Zorko, J. Dolinšek, and H. Berger, Appl. Magn. Reson. 29, 261 (2005).

${ }^{44}$ C. A. Swenson, Rev. Sci. Instrum. 68, 1312 (1997).

${ }^{45}$ A. L. R. Dawson and D. H. Ryan, Rev. Sci. Instrum. 67, 2648 (1996).

${ }^{46}$ P. Flubacher, A. J. Leadbetter, and J. A. Morrison, Philos. Mag. 4, 273 (1959).

${ }^{47}$ R. Q. Fugate and C. A. Swenson, J. Appl. Phys. 40, 3034 (1969).

${ }^{48} \mathrm{By}$ assuming ${ }^{4} \mathrm{He}$ is an ideal gas, the relationship of $C=5 / 2 R$ gives us $173.9 \mathrm{~J} \mathrm{~K}^{-1} \mathrm{~m}^{-3}$ at $2.5 \mathrm{mbar}$ and $4 \mathrm{~K}$, where $R$ is the gas constant. 
${ }^{49}$ M. Ivanda and D. Djurek, J. Phys. E 22, 988 (1989).

${ }^{50}$ Y. Kohama, T. Tojo, H. Kawaji, T. Atake, S. Matsuishi, and H. Hosono, Chem. Phys. Lett. 421, 558 (2006)

${ }^{51}$ G. A. Jorge, C. Capan, F. Ronning, M. Jaime, M. Kenzelmann, G. Gasparovic, C. Broholm, A. Ya. Shapiro, and L. A. Demianets, Physica B 354, 297 (2004).
${ }^{52}$ L. E. Svistov, A. I. Smirnov, L. A. Prozorova, O. A. Petrenko, A. Micheler, N. Büttgen, A. Ya. Shapiro, and L. N. Demianets, Phys. Rev. B 74, 024412 (2006).

${ }^{53}$ A. I. Smirnov, H. Yashiro, S. Kimura, M. Hagiwara, Y. Narumi, K. Kindo, A. Kikkawa, K. Katsumata, A. Ya. Shapiro, and L. N. Demianets, Phys. Rev. B 75, 134412 (2007). 